\title{
Coupling Poisson and Jacobi structures on foliated manifolds
}

\author{
by \\ Izu Vaisman
}

\begin{abstract}
Let $M$ be a differentiable manifold endowed with a foliation $\mathcal{F}$. A Poisson structure $P$ on $M$ is $\mathcal{F}$-coupling if $\sharp_{P}(\operatorname{ann}(T \mathcal{F}))$ is a normal bundle of the foliation. This notion extends Sternberg's coupling symplectic form of a particle in a Yang-Mills field [11. In the present paper we extend Vorobiev's theory of coupling Poisson structures [16] from fiber bundles to foliated manifolds and give simpler proofs of Vorobiev's existence and equivalence theorems of coupling Poisson structures on duals of kernels of transitive Lie algebroids over symplectic manifolds. Then we discuss the extension of the coupling condition to Jacobi structures on foliated manifolds.
\end{abstract}

The symplectic structure that describes the coupling of a particle and a field was discovered by S. Sternberg 11] (for more details, see 3]). In [16 Sternberg's symplectic form is extended to a general notion of a coupling Poisson structure on a fiber bundle and the coupling Poisson structures are used to get information about any Poisson structure in the neighborhood of a symplectic leaf. In the present paper we reprove Vorobiev's results in the context of foliated manifolds and we extend the coupling condition to Jacobi structures. In particular, we give simpler proofs for Vorobiev's existence and equivalence theorems of coupling Poisson structures on duals of kernels of transitive Lie algebroids over symplectic manifolds. The motivation for studying Poisson and Jacobi structures on a foliated manifold comes from

*2000 Mathematics Subject Classification: 53D1\%.

Key words and phrases: Foliated manifold. Coupling Poisson structure. Coupling Jacobi structure. 
the fact that a foliated manifold may play the role of the phase space of a physical system with gauge parameters, the latter being the coordinates along the leaves.

Acknowledgement. The author is grateful to Yurii Vorobiev for useful remarks during the work on this paper.

\section{Preliminaries}

Our framework is the $C^{\infty}$ category. Let $M^{m}$ be an $m$-dimensional differentiable manifold and $\mathcal{F}^{p}$ a regular foliation with $p$-dimensional leaves on $M(0 \leq p \leq m)$. We denote by $\Gamma$ spaces of global cross sections of vector bundles and by

$$
\mathcal{V}^{k}(M)=\Gamma \wedge^{k} T M, \quad \Omega^{k}(M)=\Gamma \wedge^{k} T^{*} M \quad(k=1, \ldots, m)
$$

the spaces of multivector fields and differential forms, respectively.

Furthermore, let us choose a $q$-dimensional, complementary subbundle $H$ of $F=T \mathcal{F}(q+p=m)$ i.e.,

$$
T M=H \oplus F .
$$

$H$ is called a normal bundle of the foliation and we will denote by $\pi_{H}, \pi_{F}$ the projections of $T M$ onto the subbundles $H, F$, respectively.

The decomposition (2) induces a natural bigrading of multivector fields and differential forms, and a corresponding decomposition of the exterior differential

$$
d=d_{1,0}^{\prime}+d_{0,1}^{\prime \prime}+\partial_{2,-1},
$$

where the indices denote the bidegree of the components in the direction of $H, F$, respectively [12].

In this section, we give some computation formulas for Schouten-Nijenhuis brackets (e.g., see 13.) on an arbitrary differentiable manifold and on a foliated manifold with a fixed decomposition (2).

Proposition 1.1 For any bivector field $P \in \mathcal{V}^{2}(M)$ one has

$$
[P, P](\alpha, \beta, \gamma)=2\left[d \gamma\left(\sharp_{P} \alpha, \sharp_{P} \beta\right)-\left(L_{\sharp_{P} \gamma} P\right)(\alpha, \beta)\right], \quad \alpha, \beta, \gamma \in \Omega^{1}(M),
$$

where $L$ denotes the Lie derivative and $\sharp_{P}: T^{*} M \rightarrow T M$ is defined by $\beta\left(\sharp_{P} \alpha\right)=P(\alpha, \beta)$. 
Proof. It would suffice to notice that the two sides of (4) are tensor fields, then check the formula for $\alpha, \beta, \gamma$ equal to differentials of local coordinates on $M$. Instead, in order to recall some more very important formulas, we will proceed as follows. For the bracket of 1-forms

$$
\{\alpha, \beta\}_{P}=i\left(\sharp_{P} \alpha\right) d \beta-i\left(\sharp_{P} \beta\right) d \alpha+d(P(\alpha, \beta)),
$$

one has the Gelfand-Dorfman formula [1]

$$
[P, P](\alpha, \beta, \gamma)=2\left\{\gamma\left(\sharp_{P}\{\alpha, \beta\}_{P}-\left[\sharp_{P} \alpha, \sharp_{P} \beta\right]\right)\right\} .
$$

If expression (5) is inserted in (6), a computation that uses the classical relation between the operators $d, i, L$ leads to (4). Q.e.d.

By polarizing formula (4) we get

Corollary 1.1 For any two bivector fields $P_{1}, P_{2} \in \mathcal{V}^{2}(M)$ one has

$$
\begin{gathered}
{\left[P_{1}, P_{2}\right](\alpha, \beta, \gamma)=d \gamma\left(\sharp_{P_{1}} \alpha, \sharp_{P_{2}} \beta\right)+d \gamma\left(\sharp_{P_{2}} \alpha, \sharp_{P_{1}} \beta\right)} \\
-\left(L_{\sharp_{P_{1}} \gamma} P_{2}\right)(\alpha, \beta)-\left(L_{\sharp_{P_{2}} \gamma} P_{1}\right)(\alpha, \beta) .
\end{gathered}
$$

Corollary 1.2 The bivector field $P \in \mathcal{V}^{2}(M)$ satisfies the Poisson condition $[P, P]=0$ iff

$$
\left(L_{\sharp_{P} \gamma} P\right)(\alpha, \beta)=d \gamma\left(\sharp_{P} \alpha, \sharp_{P} \beta\right) \text {. }
$$

Remark 1.1 In [1, one also finds the following formula:

$$
\begin{gathered}
\sum_{C y c l(\alpha, \beta, \gamma)}<\{\{\alpha, \beta\}, \gamma\}, X>=\left[P, L_{X} P\right](\alpha, \beta, \gamma) \\
+\frac{1}{2} \sum_{C y c l(\alpha, \beta, \gamma)}[P, P](\alpha, \beta, d<\gamma, X>), \quad \alpha, \beta, \gamma \in \Omega^{1}(M), X \in \mathcal{V}^{1}(M) .
\end{gathered}
$$

Formulas (6) and (9) show that the cotangent bundle $T^{*} M$ of a Poisson manifold $(M, P)$ with the bracket (5) is a Lie algebroid of anchor $\sharp_{P}$, a fact which is of fundamental importance in Poisson geometry. 
Now, assume the bivector field $P \in \mathcal{V}^{2}(M)$ is regular, i.e., $s=\operatorname{rank} P=$ const., and chose a decomposition

$$
T M=E \oplus D, T^{*} M=E^{*} \oplus D^{*}\left(E^{*}=\operatorname{ann} D, D^{*}=\operatorname{ann} E\right),
$$

where $D=i m \sharp_{P}$ and we have corresponding natural projections $p_{E}, p_{D}$. Then, we get an isomorphism $\sharp_{P}:$ ann $E \rightarrow D$ (by ann we denote the annihilator of a vector bundle), with an inverse $-b_{P}: D \rightarrow$ ann $E$, and there exists a well defined differential 2-form of rank $s, \theta \in \Gamma \wedge^{2} D^{*}$, defined by

$$
\theta(X, Y)=P\left[b_{P}\left(p_{D} X\right), b_{P}\left(p_{D} Y\right)\right] .
$$

Conversely, (11) allows us to reconstruct $P$ from $\theta$, such that $k e r \sharp_{P}=$ ann $D$. We will say that $\theta$ is equivalent to $P$ modulo $E$.

Proposition 1.2 For a regular bivector field $P,[P, P](\alpha, \beta, \gamma)=0$ if at least two of the arguments belongs to ann $D$, and

$$
\begin{aligned}
& {[P, P](\alpha, \beta, \gamma)=2 \gamma\left(\left[\sharp_{P} \alpha, \sharp_{P} \beta, \quad \alpha, \beta \in \text { ann } E, \gamma \in \text { ann } D\right.\right.} \\
& {[P, P](\alpha, \beta, \gamma)=2 d \theta\left(\sharp_{P} \alpha, \sharp_{P} \beta, \sharp_{P} \gamma\right), \quad \alpha, \beta, \gamma \in \text { ann } E .}
\end{aligned}
$$

Proof. We use the decomposition (10). If not all the arguments are in ann $E$ the result immediately follows from either (4) or the following GelfandDorfman expression of the Schouten-Nijenhuis bracket of two bivector fields [1]

$$
[P, P](\alpha, \beta, \gamma)=2 \sum_{C y c l(\alpha, \beta, \gamma)}<\gamma, \sharp_{P}\left(L_{\sharp P} \beta\right)>.
$$

For arguments in ann $E$, we have $\alpha=b_{P} X, \beta=b_{P} Y, \gamma=b_{P} Z$, with $X, Y, Z \in$ $\Gamma D$, and (13) yields

$$
\begin{aligned}
& {[P, P](\alpha, \beta, \gamma)=-2 \sum_{\operatorname{Cycl}(\alpha, \beta, \gamma)}<L_{X} \beta, Z>} \\
& =2 \sum_{\operatorname{Cycl}(X, Y, Z)}\{X(\theta(Y, Z))-\theta([X, Y], Z)\} .
\end{aligned}
$$

Q.e.d. 
Corollary 1.3 The regular bivector field $P$ is Poisson iff the distribution $D=i m \sharp_{P}$ is involutive and there exists a decomposition $T M=E \oplus D$ such that the equivalent to $P$, mod. E, 2-form $\theta$ satisfies the condition $\left.d \theta\right|_{D}=0$, and, then, the same holds for any such decomposition of TM.

In the case of a foliated manifold $(M, \mathcal{F})$ with a normal bundle $H$, if $P \in \mathcal{V}^{2}(M)$ is an arbitrary bivector field, it has a decomposition

$$
P=P_{2,0}^{\prime}+\bar{P}_{1,1}+P_{0,2}^{\prime \prime},
$$

and we can compute the corresponding decomposition of the Schouten-Nijenhuis bracket $[P, P]$ by applying formula (44) to 1 -forms $\alpha, \beta, \gamma \in \Omega^{1,0}(M)$ and $\lambda, \mu, \nu \in \Omega^{0,1}(M)$. The results are contained in the following formulas

$$
\begin{aligned}
& {\left[P^{\prime}, P^{\prime}\right]_{3,0}(\alpha, \beta, \gamma)=2\left[d^{\prime} \gamma\left(\sharp_{P^{\prime}} \alpha, \sharp_{P^{\prime}} \beta\right)-\left(L_{\sharp_{P^{\prime}}} P^{\prime}\right)(\alpha, \beta)\right] \text {, }} \\
& {\left[P^{\prime}, P^{\prime}\right]_{2,1}(\alpha, \beta, \lambda)=2 \partial \lambda\left(\sharp_{P^{\prime}} \alpha, \sharp_{P^{\prime}} \beta\right)=-2 \lambda\left(\left[\sharp_{P^{\prime}} \alpha, \sharp_{P^{\prime}} \beta\right]\right) \text {, }} \\
& {\left[P^{\prime}, P^{\prime}\right]_{1,2}(\alpha, \lambda, \mu)=0, \quad\left[P^{\prime}, P^{\prime}\right]_{0,3}(\lambda, \mu, \nu)=0,} \\
& {[\bar{P}, \bar{P}]_{3,0}(\alpha, \beta, \gamma)=0 \text {, }} \\
& {[\bar{P}, \bar{P}]_{2,1}(\alpha, \beta, \lambda)=2\left[d^{\prime \prime} \lambda\left(\sharp_{\bar{P}} \alpha, \sharp_{\bar{P}} \beta\right)-\left(L_{\sharp_{\bar{P}} \lambda} \bar{P}\right)(\alpha, \beta)\right] \text {, }} \\
& {[\bar{P}, \bar{P}]_{1,2}(\alpha, \lambda, \mu)=-2\left[d^{\prime} \mu\left(\sharp_{\bar{P}} \lambda, \sharp_{\bar{P}} \alpha\right)+\left(L_{\sharp_{\bar{P}}} \bar{P}\right)(\alpha, \lambda)\right] \text {, }} \\
& {[\bar{P}, \bar{P}]_{0,3}(\lambda, \mu, \nu)=2\left[\partial \nu\left(\sharp_{\bar{P}} \lambda, \sharp_{\bar{P}} \mu\right)-\left(L_{\sharp_{\bar{P}} \nu} \bar{P}\right)(\lambda, \mu)\right] \text {, }} \\
& {\left[P^{\prime \prime}, P^{\prime \prime}\right]_{3,0}(\alpha, \beta, \gamma)=0, \quad\left[P^{\prime \prime}, P^{\prime \prime}\right]_{2,1}(\alpha, \beta, \lambda)=0,} \\
& {\left[P^{\prime \prime}, P^{\prime \prime}\right]_{1,2}(\alpha, \lambda, \mu)=0 \text {, }} \\
& {\left[P^{\prime \prime}, P^{\prime \prime}\right]_{0,3}(\lambda, \mu, \nu)=2\left[d^{\prime \prime} \nu\left(\sharp_{P^{\prime \prime}} \lambda, \sharp_{P^{\prime \prime}} \mu\right)-\left(L_{\sharp_{P^{\prime \prime}} \nu} P^{\prime \prime}\right)(\lambda, \mu)\right] \text {, }} \\
& {\left[P^{\prime}, \bar{P}\right]_{3,0}(\alpha, \beta, \gamma)=d^{\prime \prime} \gamma\left(\sharp_{P^{\prime}} \alpha, \sharp_{\bar{P}} \beta\right)-d^{\prime \prime} \gamma\left(\sharp_{P^{\prime}} \beta, \sharp_{\bar{P}} \alpha\right)} \\
& -\left(L_{\sharp_{P^{\prime}} \gamma} \bar{P}\right)(\alpha, \beta)-\left(L_{\sharp_{\bar{P}} \gamma} P^{\prime}\right)(\alpha, \beta), \\
& {\left[P^{\prime}, \bar{P}\right]_{2,1}(\alpha, \beta, \lambda)=d^{\prime} \lambda\left(\sharp_{P^{\prime}} \alpha, \sharp_{\bar{P}} \beta\right)-d^{\prime} \lambda\left(\sharp_{P^{\prime}} \beta, \sharp_{\bar{P}} \alpha\right)} \\
& -\left(L_{\sharp_{\bar{P}} \lambda} P^{\prime}\right)(\alpha, \beta), \\
& {\left[P^{\prime}, \bar{P}\right]_{1,2}(\alpha, \lambda, \mu)=\partial \mu\left(\sharp_{P^{\prime}} \alpha, \sharp_{\bar{P}} \lambda\right)-\left(L_{\sharp_{\bar{P}} \mu} P^{\prime}\right)(\alpha, \lambda)} \\
& =-\left(L_{\sharp_{P^{\prime}} \alpha} \bar{P}\right)(\lambda, \mu), \quad\left[P^{\prime}, \bar{P}\right]_{0,3}(\lambda, \mu, \nu)=0,
\end{aligned}
$$




$$
\begin{aligned}
& {\left[P^{\prime}, P^{\prime \prime}\right]_{3,0}(\alpha, \beta, \gamma)=0, \quad\left[P^{\prime}, P^{\prime \prime}\right]_{0,3}(\lambda, \mu, \nu)=0,} \\
& {\left[P^{\prime}, P^{\prime \prime}\right]_{2,1}(\alpha, \beta, \lambda)=-\left(L_{\sharp_{P^{\prime \prime}} \lambda} P^{\prime}\right)(\alpha, \beta)} \\
& {\left[P^{\prime}, P^{\prime \prime}\right]_{1,2}(\alpha, \lambda, \mu)=d^{\prime} \mu\left(\sharp_{P^{\prime}} \alpha, \sharp_{P^{\prime \prime}} \lambda\right)-\left(L_{\sharp_{P^{\prime \prime}} \mu} P^{\prime}\right)(\alpha, \lambda)} \\
& =\left(L_{\sharp_{P^{\prime}} \alpha} P^{\prime \prime}\right)(\lambda, \mu), \\
& {\left[\bar{P}, P^{\prime \prime}\right]_{3,0}(\alpha, \beta, \gamma)=0, \quad\left[\bar{P}, P^{\prime \prime}\right]_{2,1}(\alpha, \beta, \lambda)=0,} \\
& {\left[\bar{P}, P^{\prime \prime}\right]_{1,2}(\alpha, \lambda, \mu)=d^{\prime \prime} \mu\left(\sharp_{\bar{P}} \alpha, \sharp_{P^{\prime \prime}} \lambda\right)-\left(L_{\sharp_{\bar{P}} \mu} P^{\prime \prime}\right)(\alpha, \lambda)} \\
& -\left(L_{\sharp_{P^{\prime \prime} \mu}} \bar{P}\right)(\alpha, \lambda), \\
& {\left[\bar{P}, P^{\prime \prime}\right]_{0,3}(\lambda, \mu, \nu)=d^{\prime} \nu\left(\sharp_{\bar{P}} \lambda, \sharp_{P^{\prime \prime}} \mu\right)-d^{\prime} \nu\left(\sharp_{\bar{P}} \mu, \sharp_{P^{\prime \prime}} \lambda\right)} \\
& -\left(L_{\sharp_{\bar{P}} \nu} P^{\prime \prime}\right)(\lambda, \mu)-\left(L_{\sharp_{P^{\prime \prime}} \nu} \bar{P}\right)(\lambda, \mu) .
\end{aligned}
$$

Finally, we indicate a way to compute the bigraded components of any Schouten-Nijenhuis bracket. Let us recall the following general formula of Lichnerowicz (e.g., see [13]):

$$
\begin{aligned}
& i([P, Q]) \varphi=(-1)^{q}(p+1) i(P) d(i(Q) \varphi) \\
& \quad+(-1)^{p} i(Q) d(i(P) \varphi)-i(P \wedge Q) d \varphi,
\end{aligned}
$$

where $P \in \mathcal{V}^{p}(M), Q \in \mathcal{V}^{q}(M)$, and $\varphi \in \Omega^{p+q-1}(M)$. The operator $i$ is the interior product. If $g$ is an arbitrary Riemannian metric on $M, i(P)$ is the transposed operator of the wedge product by $b_{g} P[\underline{6}$, whence, it follows that

$$
i(P \wedge Q)=i(Q) \circ i(P)
$$

Now, if $P$ is of bidegree $(a, b)(a+b=p)$ and $Q$ is of bidegree $(h, k)$ $(h+k=q)$, the component $[P, Q]^{u, v}(u+v=p+q-1)$ is provided by using (21) for $\varphi \in \Omega^{u, v}(M)$. With (3), we see that the only possibilities to get non-zero components correspond to the replacement of $d$ by $d^{\prime}, d^{\prime \prime}, \partial$ in (21), which leads to the cases

$$
\begin{gathered}
u=a+h-1, v=b+k ; u=a+h, v=b+k-1 ; \\
u=a+h-2, v=b+k+1 .
\end{gathered}
$$

All the other components vanish because of degree incompatibility. 


\section{Leaf-tangent Poisson structures}

In this section we discuss Poisson structures along the leaves of a foliation since these will be a basic ingredient of the coupling Poisson structures. The notation is that of Section 1.

Definition 2.1 The Poisson structure defined by the bivector field $P \in$ $\mathcal{V}^{2}(M)$ is leaf-tangent to $\mathcal{F}$ if its symplectic leaves are submanifolds of the leaves of $\mathcal{F}$, equivalently, if the leaves of $\mathcal{F}$ are Poisson submanifolds of $(M, P)$ $13]$.

Obviously, $P$ is $\mathcal{F}$-leaf-tangent iff $P \in \Gamma \wedge^{2} F(F=T \mathcal{F})$, which is equivalent with the fact that, for all the choices of a normal bundle $H$ satisfying (2), the decomposition (14) of $P$ satisfies the conditions

$$
P_{1,1}^{\prime}=0, \quad \bar{P}_{1,2}=0 .
$$

Proposition 2.1 A bivector field $P \in \Gamma \wedge^{2} F$ is a Poisson bivector field on $M$ iff

$$
d^{\prime \prime} \nu\left(\sharp_{P} \lambda, \sharp_{P} \mu\right)-\left(L_{\sharp} \nu P\right)(\lambda, \mu)=0, \quad \forall \lambda, \mu, \nu \in \Omega^{0,1}(M),
$$

equivalently, iff the restrictions of $P$ to the leaves are Poisson bivector fields of the leaves.

Proof. $P$ is Poisson iff $[P, P]=0$ and, by (15)-(20), this condition reduces to (25). Q.e.d.

Therefore, a leaf-tangent Poisson structure may be identified with a family $P_{L}$ of Poisson structures of the leaves $L$ of $\mathcal{F}$ such that, $\forall f, g \in C^{\infty}(M)$, the function

$$
\{f, g\}(x)=\left\{\left.f\right|_{L(x)},\left.g\right|_{L(x)}\right\}_{\left.P\right|_{L(x)}}(x),
$$

where $x \in M$ and $L(x)$ is the leaf of $\mathcal{F}$ through $x$, also belongs to $C^{\infty}(M)$.

It is worthwhile noticing that, if $P$ is $\mathcal{F}$-leaf-tangent, $\mathcal{F}$ may be seen as a regularizing foliation of the symplectic foliation $\mathcal{S}$ of $P$ (usually, $\mathcal{S}$ has singular points). This idea leads to a global, numerical invariant of a Poisson structure on a manifold $M^{m}$, the regularizing dimension, to be defined as the smallest possible dimension $p \leq m$ of a regularizing foliation. 
Example 2.1 Put $\mathbb{R}^{m}=\mathbb{R}^{3} \times \mathbb{R}^{m-3}$ and take the Poisson structure $P$ defined by the Lie-Poisson structure of the factor $\mathbb{R}^{3}$ seen as the Lie algebra $s o(3)$ (e.g., [13]). $P$ is leaf-tangent to the foliation defined by the factor $\mathbb{R}^{3}$ of $\mathbb{R}^{m}$, and the regularizing dimension of $P$ is 3 .

Example 2.2 Consider the compact nilmanifold $M(1, n)=\Gamma(1, n) \backslash H(1, n)$ where

$$
H(1, n)=\left\{\left(\begin{array}{ccc}
I d_{n} & X & Z \\
0 & 1 & y \\
0 & 0 & 1
\end{array}\right) / X, Z \in \mathbb{R}^{n}, y \in \mathbb{R}\right\}
$$

is the generalized Heisenberg group, and $\Gamma(1, n)$ is the subgroup of matrices with integer entries. $M(1, n)$ has a natural atlas with the transition functions

$$
\tilde{x}^{i}=x^{i}+a^{i}, \tilde{y}=y+b, \tilde{z}^{i}=z^{i}+a^{i} y+c^{i},
$$

where $x^{i}, z^{i}(i=1, \ldots, n)$ are the entries of $X, Z$, respectively, and $a^{i}, b, c^{i}$ are integers, and it is parallelizable by the global vector fields

$$
\frac{\partial}{\partial x^{i}}, \frac{\partial}{\partial y}+\sum_{i=1}^{p} x^{i} \frac{\partial}{\partial z^{i}}, \frac{\partial}{\partial z^{i}}
$$

It follows that

$$
P=\sin (2 \pi y) \frac{\partial}{\partial x^{1}} \wedge \frac{\partial}{\partial z^{1}}
$$

yields a Poisson structure of $M(1, n)$ which is leaf-tangent to the $(n+1)$ dimensional foliation

$$
y=\text { const. }, x^{t}=\text { const } . \quad(t=2, \ldots, n) .
$$

But, $P$ is also leaf-tangent to the 2-dimensional foliation $\operatorname{span}\left\{\partial / \partial x^{1}, \partial / \partial z^{1}\right\}$, and the regularizing dimension of $P$ is 2 .

Example 2.3 [16] Let $p: \mathbb{G}^{*} \rightarrow B$ be a bundle of Lie coalgebras (i.e., the dual of a bundle $p: \mathbb{G} \rightarrow B$ of Lie algebras) over a manifold $B$. Then the Lie-Poisson structures of the fibers yield a leaf-tangent Poisson structure $\mathbb{L}$ 
of $\mathbb{G}^{*}$. If $\left(x^{i}\right)$ are local coordinates on $B$ and $\left(y_{a}\right)$ are linear coordinates along the fibers of $\mathbb{G}^{*}$, one has 13 .

$$
\mathbb{L}=\frac{1}{2} \alpha_{a b}^{c}(x) y_{c} \frac{\partial}{\partial y_{a}} \wedge \frac{\partial}{\partial y_{b}},
$$

where the Einstein summation convention is used and $\alpha_{a b}^{c}(x)$ are the structural constants of the corresponding fibers of $\mathbb{G}$. The invariant expression of $\mathbb{L}$ on differentials at $z \in \mathbb{G}^{*}$ of fiberwise linear functions on $\mathbb{G}^{*}$, seen as elements $X, Y \in \mathbb{G}_{p(z)}$, is

$$
\mathbb{L}_{z}(X, Y)=<z, C_{p(z)}(X, Y)>,
$$

where the "tensor field" $C \in \Gamma\left[\left(\wedge^{2} \mathbb{G}^{*}\right) \otimes(\mathbb{G})\right]$ is defined by

$$
C_{p(z)}(X, Y)=[X, Y]_{\mathbb{G}_{p(z)}} .
$$

The properties of a leaf-tangent Poisson structure reflect corresponding properties along the leaves. An interesting situation appears for the Poisson cohomology.

Proposition 2.2 Let $P$ be a leaf-tangent Poisson structure on $(M, \mathcal{F})$ and let $\sigma$ be the Lichnerowicz coboundary operator of $P$. Then, for every normal bundle $H$ of $\mathcal{F}$, one has a decomposition

$$
\sigma=\sigma_{-1,2}^{\prime}+\sigma_{0,1}^{\prime \prime}
$$

where the terms are homogeneous components of $\sigma$ of indicated bidegree and

$$
\sigma^{2}=0, \sigma^{\prime \prime 2}=0, \sigma^{\prime} \circ \sigma^{\prime \prime}+\sigma^{\prime \prime} \circ \sigma^{\prime}=0
$$

Proof. The Lichnerowicz coboundary operator is $\sigma Q=-[P, Q], \forall Q \in$ $\mathcal{V}^{*}(M)$ (e.g., 13]). From (23), since $P$ is homogeneous of bidegree $(0,2)$, it follows that $\sigma$ may have only components of bidegree $(-1,2),(0,1)$ and $(-2,3)$. We will prove that the $(-2,3)$-component of $\sigma$ vanishes.

Indeed, $\sigma_{-2,3}$ is obtained by computing $i([P, Q]) \varphi, Q \in \mathcal{V}^{h, k}(M), \varphi \in$ $\Omega^{h-2, k+3}$ via (21). Since in this case $i(Q) \varphi=0$ (it should be of bidegree $(-2,3))$, using (22) we get

$$
i([P, Q]) \varphi=i(Q)[d i(P) \varphi-i(P) d \varphi]
$$


where, in fact, only the component $\partial$ of $d$ may bring a non zero contribution.

Now, let us evaluate a Lie derivative $L_{Y} \psi$ where $Y \in \Gamma F$ and $\psi \in \Omega^{s, t}(M)$ for arguments $X_{1}, \ldots, X_{u} \in \mathcal{V}_{p r}^{1,0}(M), Y_{1}, \ldots, Y_{v} \in \mathcal{V}^{0,1}(M)$, where $\mathcal{V}_{p r}^{1,0}(M)$ is the subspace of the $(1,0)$-vector fields that project onto the space of leaves of $\mathcal{F}$, which means that, $\forall Y \in \Gamma F,\left[Y, X_{i}\right] \in \Gamma F(i=1, \ldots, u)$. (It is enough to use only this kind of arguments $X$ because $L_{Y} \psi$ is a tensor, hence, its values at each point depend on the values of the arguments at that point only.) We have

$$
\begin{gathered}
\left(L_{Y} \psi\right)\left(X_{1}, \ldots, X_{u}, Y_{1}, \ldots, Y_{v}\right)=Y\left(\psi\left(X_{1}, \ldots, X_{u}, Y_{1}, \ldots, Y_{v}\right)\right) \\
-\sum_{i=1}^{u} \psi\left(X_{1}, \ldots, X_{i-1},\left[Y, X_{i}\right], X_{i+1}, \ldots, X_{u}, Y_{1}, \ldots, Y_{v}\right) \\
-\sum_{j=1}^{v} \psi\left(X_{1}, \ldots, X_{u}, Y_{1}, \ldots, Y_{j-1},\left[Y, Y_{j}\right], Y_{j+1}, \ldots, Y_{v}\right)=0
\end{gathered}
$$

and it follows that $L_{Y} \psi$ may have only components of bidegree equal either to $(s, t)$ or to $(s-1, t+1)$.

From the previous remark, it follows that, $\forall Q \in \mathcal{V}^{h, k}(M), \forall \psi \in \Omega^{h-2, k+3} \oplus$ $\Omega^{h-2, k+2}$ and $\forall Y \in \Gamma F$, one has

$$
i(Q) L_{Y} \psi=i(Q)[d i(Y)+i(Y) d] \psi=0 .
$$

Furthermore, for $\varphi \in \Omega^{h-2, k+3}$, (22) and (37) imply

$$
\begin{gathered}
i(Q)\left[i\left(Y_{1} \wedge Y_{2}\right) d-d i\left(Y_{1} \wedge Y_{2}\right)\right] \varphi=i\left(Y_{2} \wedge Q\right) i\left(Y_{1}\right) d \varphi-i(Q) d i\left(Y_{2}\right) i\left(Y_{1}\right) \varphi \\
=-i\left(Y_{2} \wedge Q\right) d i\left(Y_{1}\right) \varphi+i(Q) i\left(Y_{2}\right) d i\left(Y_{1}\right) \varphi=0 .
\end{gathered}
$$

Since $P$ is spanned over $\mathbb{R}$ by wedge products $Y_{1} \wedge Y_{2}$ of vector fields tangent to $\mathcal{F}$, the expression given by (36) vanishes.

The properties (35) follow from $\sigma^{2}=0$. Q.e.d.

Even though we have (35), the degrees are not right for a double cochain complex. However, $\left(\mathcal{W}^{h, k}=\mathcal{V}^{k, h}(M), \sigma\right)$ is a double, semipositive, cochain complex, and the cohomology of such a complex is the limit of a spectral sequence [12. More exactly, we have

Proposition 2.3 Let $P$ be a leaf-tangent Poisson structure on $(M, \mathcal{F})$ and denote by $H_{L P}^{h}(\mathcal{F}, P)$ the cohomology spaces of the cochain complex $\left(\mathcal{V}^{0, *}, \sigma^{\prime \prime}\right)$. 
Then, the Poisson cohomology of $P$ is the limit of a spectral sequence $\left(E_{r}^{h k}, d_{r}\right)$ where

$$
E_{2}^{h k}=\mathcal{V}^{k, 0}(M) \otimes_{\mathbb{R}} H_{L P}^{h}(\mathcal{F}, P)
$$

and $d_{2}$ is induced by the operator $\sigma^{\prime}$.

Proof. The spaces

$$
\mathcal{W}_{l}(M)=\oplus_{k \geq l} \oplus_{h} \mathcal{V}^{h k}(M)
$$

yield a regular filtration of the Lichnerowicz-Poisson complex $(\mathcal{V}(M), \sigma)$ and the required spectral sequence is the spectral sequence $\left(E_{r}^{h k}, d_{r}\right)$ defined by this filtration. From the definition of a spectral sequence we get

$$
E_{0}^{k h}=\mathcal{V}^{h, k}(M), \quad d_{0}=0 .
$$

The cohomology of $E_{0}$ yields

$$
E_{1}^{k h}=\mathcal{V}^{h, k}(M), \quad d_{1}=\tau^{\prime \prime} .
$$

Finally, the cohomology spaces of the complex $E_{1}$ are the spaces given by formula (38) and $d_{2}$ is induced by $\tau^{\prime}$. Q.e.d.

Remark 2.1 The leaf-tangent Poisson bivector field $P \in \Gamma \wedge^{2} F$ may be seen as a Poisson bivector of the Lie algebroid $F$ defined by the foliation. As such, it induces a Lie algebroid structure on the dual bundle $F^{*}$, and $H_{L P}^{h}(\mathcal{F}, P)$ are the cohomology spaces of this Lie algebroid (e.g., [] $]$ ).

\section{Coupling Poisson structures}

In this section we present the general results concerning coupling Poisson structures on foliated manifolds. Again, the notation is that of Section 1, we consider the manifold $M$, the foliation $\mathcal{F}$, the normal bundle $H$, and the bivector field $P$ written under the form (14).

Definition 3.1 The bivector field $P$ is $\mathcal{F}$-almost coupling via $H$ if, $\forall x \in M$,

$$
\sharp_{P}\left(\operatorname{ann} F_{x}\right) \subseteq H_{x} \text {. }
$$

ii) The bivector field $P$ is $\mathcal{F}$-coupling if $\sharp_{P}($ ann $F)$ is a normal bundle $H$ of $\mathcal{F}$. In both cases, if $P$ is Poisson, the coupling property is attributed to the Poisson structure. 
With (14), it follows that the almost coupling condition is equivalent with the vanishing of the $(1,1)$-component $\bar{P}=0$, hence, also with $\sharp_{P}($ ann $H) \subseteq$ $F$. The coupling condition is equivalent with

$$
\operatorname{dim}\left(\sharp_{P}(\operatorname{ann} F)\right)=q,
$$

the codimension of $\mathcal{F}$. The first term of (40) is the rank of the term $P^{\prime}$ of (14) for any choice of $H$, hence, coupling may exist only if $q$ is even. In the case of a coupling field $P$, we always shall take $H=\sharp_{P}($ ann $F)$.

Example 3.1 Consider again the manifold $M(1, n)$ of Example 2.2. With the notation of that example, define the foliation $\mathcal{F}$ by the tangent distribution

$$
F=\operatorname{span}\left\{\frac{\partial}{\partial x^{1}}, \frac{\partial}{\partial z^{1}}, \frac{\partial}{\partial y}+\sum_{i=1}^{n} x^{i} \frac{\partial}{\partial z^{i}}\right\}
$$

and the Poisson bivector field

$$
P=\sum_{t=2}^{n} \frac{\partial}{\partial x^{t}} \wedge \frac{\partial}{\partial z^{t}}+\sin 2 \pi y \frac{\partial}{\partial x^{1}} \wedge \frac{\partial}{\partial z^{1}} .
$$

Obviously, $P$ is $\mathcal{F}$-coupling.

Example 3.2 Let $P$ be an arbitrary Poisson bivector field on the foliated manifold $(M, \mathcal{F})$. Assume that there exists a symplectic leaf $S$ of $P$ which is embedded in $M$ and transversal to $\mathcal{F}$. (Generally, such a situation may appear on an open subset $U$ of $M$.) Then condition (40) holds on $S$, therefore, also on some open neighborhood $V$ of $S$, and $P$ is $\mathcal{F}$-coupling on $V$. In particular, for any Poisson structure $P$ and any symplectic leaf $S$ of $P$ which is embedded in $M$, there exists a tubular neighborhood $V$ of $S$ where $P$ is coupling with respect to the fibers of the tubular structure of $V$. This remark is due to Vorobiev [16].

Proposition 3.1 An almost coupling bivector field $P$ is Poisson iff

$$
\begin{aligned}
& d^{\prime} \gamma\left(\sharp_{P^{\prime}} \alpha, \sharp_{P^{\prime}} \beta\right)-\left(L_{\sharp_{P^{\prime}} \gamma} P^{\prime}\right)(\alpha, \beta)=0, \\
&\left(L_{\sharp_{P^{\prime \prime}} \lambda} P^{\prime}\right)(\alpha, \beta)+\lambda\left(\left[\sharp_{P^{\prime}} \alpha, \sharp_{P^{\prime}} \beta\right]\right)=0, \\
&\left(L_{\sharp_{P^{\prime}} \alpha} P^{\prime \prime}\right)(\lambda, \mu)=0, \\
& d^{\prime \prime} \nu\left(\sharp_{P^{\prime \prime}} \lambda, \sharp_{P^{\prime \prime}} \mu\right)-\left(L_{\sharp_{P^{\prime \prime}} \nu} P^{\prime \prime}\right)(\lambda, \mu)=0, \\
& \forall \alpha, \beta, \gamma \in \Omega^{1,0}(M), \forall \lambda, \mu, \nu \in \Omega^{0,1}(M) .
\end{aligned}
$$


Proof. Use (15)-(20) to express $[P, P]=0$ for

$$
P=P^{\prime}+P^{\prime \prime} \text {. }
$$

Q.e.d.

The last condition (43) is (25), again, and it means that the component $P^{\prime \prime}$ is an $\mathcal{F}$-leaf-tangent Poisson bivector field.

In the coupling case, the Poisson condition may be put in the form given by Vorobiev [16] for fiber bundles.

Proposition 3.2 A coupling Poisson structure of a foliated manifold $(M, \mathcal{F})$ is equivalent with a triple $\left(P^{\prime \prime}, H, \sigma\right)$, where $P^{\prime \prime}$ is a leaf-tangent Poisson structure, $H$ is a normal bundle of $\mathcal{F}$ and $\sigma$ is a non-degenerate cross section of $\wedge^{2}($ ann $F)$ such that

$$
\begin{gathered}
d^{\prime} \sigma=0, \\
\sharp_{P^{\prime \prime}}\{d[\sigma(X, Y)]\}=-p_{F}[X, Y], \quad \forall X, Y \in \mathcal{V}_{p r}^{1,0}(M), \\
L_{X} P^{\prime \prime}=0, \quad \forall X \in \mathcal{V}_{p r}^{1,0}(M) .
\end{gathered}
$$

Proof. Let $P$ be a coupling Poisson bivector field written under the form (44). The coupling condition defines $H$ and $P^{\prime \prime}$, and the last condition (43) is equivalent with the fact that $P^{\prime \prime}$ is a Poisson bivector field. Furthermore, $P^{\prime}$ is a regular bivector field with $i m \sharp_{P^{\prime}}=H$, and we may write $P^{\prime}=\sharp_{P^{\prime}}(\sigma)$ $=\sharp_{P}(\sigma)$, where $\sigma \in \wedge^{2}($ ann $F)$ is equivalent with $P^{\prime}$ mod. $F$ and has the maximal rank. Accordingly, for the 1 -forms of bidegree $(1,0)$ of (43) we may write

$$
\alpha=b_{\sigma} X, \beta=b_{\sigma} Y, \gamma=b_{\sigma} Z, \quad X, Y, Z \in \mathcal{V}^{1,0}(M),
$$

where $X, Y, Z$ are uniquely defined. With this representation, formulas (4), (12) show that the first condition (43) becomes (45).

Conditions (43) are tensorial. Thus, in particular, the second condition (43) holds iff it holds for $X, Y \in \mathcal{V}_{p r}^{1,0}(M)$ in (48). In this case, using the definition of the Lie derivative, we see that the second condition (43) becomes (46). 
Finally, the third condition (43) is equivalent to (47) because, for a projectable vector field $X, L_{X} P^{\prime \prime}$ vanishes if at least one of its arguments is of bidegree $(1,0)$.

For the converse, first notice that we may define a triple $\left(P^{\prime \prime}, H, \sigma\right)$ for any coupling bivector field $P$. The 2 -form $\sigma$ will be called a coupling form of the leaf-tangent bivector field $P^{\prime \prime}$ and one has an isomorphism $b_{\sigma}: H \rightarrow$ ann $F$. Then, we can reconstruct $P$ from such a triple by the formula

$$
P(\xi, \eta)=\sigma\left(b_{\sigma}^{-1} \xi^{\prime}, b_{\sigma}^{-1} \eta^{\prime}\right)+P^{\prime \prime}\left(\xi^{\prime \prime}, \eta^{\prime \prime}\right),
$$

where

$$
\xi=\xi^{\prime}+\xi^{\prime \prime}, \eta=\eta^{\prime}+\eta^{\prime \prime}, \quad \xi^{\prime}, \eta^{\prime} \in \operatorname{ann} F, \xi^{\prime \prime}, \eta^{\prime \prime} \in \operatorname{ann} H .
$$

If we reconstruct $P$ from $\left(P^{\prime \prime}, H, \sigma\right)$ that satisfy (45), (46), (47), and where $P^{\prime \prime}$ is Poisson, conditions (43) will hold. Q.e.d.

Remark 3.1 If the triple $\left(P^{\prime \prime}, H, \sigma\right)$ satisfies all the conditions of Proposition 3.2. these conditions are also satisfied by any triple $\left(P^{\prime \prime}, H, \sigma+\epsilon \tau\right)$ where $\tau \in$ $\wedge^{2}(\operatorname{ann} F)$ is closed and $\epsilon \in \mathbb{R}$. If $\epsilon$ is small enough, $\sigma+\epsilon \tau$ is non degenerate on $H$, and the new triple also provides a coupling Poisson structure.

Remark 3.2 If the coupling Poisson tensor field $P$ of (44) is defined by a symplectic form $\omega$ of a manifold $M, P^{\prime \prime}$ is equivalent with a closed 2-form $\theta$ of bidegree $(0,2)$ that defines a symplectic structure on each leaf of $\mathcal{F}$, and the form $\sigma$ of the corresponding triple is a corresponding coupling form in the sense of [3].

We also notice the following, rather clear, assertion

Proposition 3.3 Two coupling bivector fields $P_{1}, P_{2}$ are equivalent by a foliated diffeomorphism $\Phi$ iff the corresponding triples are equivalent by $\Phi$.

Proof. By equivalence we mean that $\Phi_{*}\left(P_{1}\right)=P_{2}$, i.e.,

$$
\sharp_{P_{2}(\phi(x))}=\Phi_{*}(x) \circ \sharp_{P_{1}(x)} \circ \Phi^{*}(\Phi(x)), \quad(x \in M),
$$

and a diffeomorphism $\Phi: M \rightarrow M$ is foliated if it preserves the foliation $\mathcal{F}$, whence, $\Phi_{*}\left(F_{x}\right)=F_{\Phi(x)}$ and $\Phi^{*}\left(\operatorname{ann} F_{\Phi(x)}\right)=\operatorname{ann} F_{x}$. These properties, and 
(50), imply that, if $P_{1}$ is coupling, $P_{2}$ necessarily is coupling as well and it defines the normal bundle $H_{2}=\Phi_{*}\left(H_{1}\right)$. Now, using the decompositions

$$
T M=H_{1} \oplus F=H_{2} \oplus F
$$

it follows that $P_{2}^{\prime \prime}=\Phi_{*}\left(P_{1}^{\prime \prime}\right)$ and $\sigma_{2}=\Phi^{*} \sigma_{1}$. Conversely, the equivalence of the triples implies that of the bivector fields in view of formula (49). Q.e.d.

Remark 3.3 A Poisson equivalence of coupling Poisson structures which is not a foliated diffeomorphism does not preserve the elements of the associated triples.

The conditions indicated in Proposition 3.2 can be expressed in a more detailed way if $P^{\prime \prime}$ is a regular bivector field.

Proposition 3.4 A regular coupling Poisson structure of a foliated manifold $(M, \mathcal{F})$ is equivalent with a class of objects $(S, G, H, \sigma, \theta)$, where $S, G, H$ are vector subbundles of $T M$ such that: i) $S$ is integrable, $F=G \oplus S$ and $T M=H \oplus F$; ii) $\sigma \in \Gamma \wedge^{2}$ (ann $\left.F\right)$ is non degenerate and satisfies the condition $d^{\prime} \sigma=0$; iii) $\theta \in \Gamma \wedge^{2}(\operatorname{ann}(H \oplus G))$ is non degenerate and satisfies the condition $\left.d \theta\right|_{S}=0$; iv) for any projectable vector field $X \in \Gamma H$ and any vector fields $U, V \in \Gamma S$ one has $[X, U] \in \Gamma S$ and $\left.\left(L_{X} \theta\right)(U, V)=0 ; v\right)$ for any projectable vector fields $X, Y \in \Gamma H$, the projection $p_{F}[X, Y] \in \Gamma S$ and $b_{\theta}\left(p_{F}[X, Y]\right)=d[\sigma(X, Y)]$, where $b_{\theta}$ is the isomorphism $S \rightarrow \operatorname{ann}(H \oplus G)$ defined by $\theta$.

Proof. Consider the associated triple $\left(P^{\prime \prime}, H, \sigma\right)$ that satisfies the conditions of Proposition [3.2, and ask $P^{\prime \prime}$ to have a constant rank. Let $S$ be the tangent bundle of the symplectic foliation of $P^{\prime \prime}$, let $G$ be an arbitrary tangent subbundle such that $F=G \oplus S$, and let $\theta$ be the equivalent 2-form of $P^{\prime \prime}$, mod. $H \oplus G$. Then formulas (41), (12) and the integrability of $S$ show that the last condition (43) is exactly $\left.d \theta\right|_{S}=0$.

As indicated in the proof of Proposition 3.2. (47) holds iff it holds for arguments $\lambda, \mu \in \Gamma($ ann $H)$, which may be further decomposed as $\lambda=\lambda_{1}+$ $\lambda_{2}, \mu=\mu_{1}+\mu_{2}$, where $\lambda_{1}, \mu_{1} \in \Gamma(\operatorname{ann}(H \oplus S)), \lambda_{2}, \mu_{2} \in \Gamma(\operatorname{ann}(H \oplus G))$. Then, $\sharp_{P^{\prime \prime}} \lambda_{1}=0, \sharp_{P^{\prime \prime}} \mu_{1}=0$, and $\lambda_{2}=b_{\theta} U, \mu_{2}=b_{\theta} V$ with $U, V \in \Gamma S$, and $\forall X \in \mathcal{V}_{p r}^{1,0}(M)$ we get

$$
\begin{gathered}
\left(L_{X} P^{\prime \prime}\right)\left(\lambda_{1}+\lambda_{2}, \mu_{1}+\mu_{2}\right)=X(\theta(V, U))+\theta(U,[X, V])-\theta(V,[X, U]) \\
+\lambda_{1}([X, V])-\mu_{1}([X, U])=-\left(L_{X} \theta\right)(U, V)+\lambda_{1}([X, V])-\mu_{1}([X, U]) .
\end{gathered}
$$


Since $\lambda_{1}, \mu_{1} \in \Gamma(\operatorname{ann}(H \oplus S))$ are arbitrary arguments, the result of (151) is zero iff condition iv) of the proposition holds.

The equivalence between (46) and condition v) is obvious.

The computations above also show that a system $(S, G, H, \sigma, \theta)$ which satisfies the conditions of the present proposition produces a triple $\left(P^{\prime \prime}, H, \sigma\right)$ that satisfies the conditions of Proposition 3.2. Two systems of data ( $S, G, H$, $\sigma, \theta)$ will be in the same class, in the sense of the proposition, if they produce the same bivector field $P^{\prime \prime}$. Q.e.d.

In particular, we get the characteristic conditions of [3] for the symplectic coupling forms:

Corollary 3.1 Let $(M, \mathcal{F})$ be a foliated manifold endowed with a symplectic form $\omega$. Then $\omega$ is $\mathcal{F}$-coupling iff the $\omega$-orthogonal distribution $H$ of $F$ is a normal bundle of $\mathcal{F}$ and one has a corresponding decomposition

$$
\omega=\sigma_{2,0}+\theta_{0,2}
$$

that satisfies the following conditions $i)$ the form $\theta$ is symplectic on each leaf of $\mathcal{F}$, and the form $\sigma$ is non degenerate with $d^{\prime} \sigma=0$; ii) $\forall X \in \mathcal{V}_{p r}^{1,0}(M)$, the form $L_{X} \theta$ vanishes on $F$, iii) $\forall X, Y \in \mathcal{V}_{p r}^{1,0}(M)$, one has

$$
b_{\theta}\left(p_{F}[X, Y]\right)=d\{\sigma(X, Y)\}
$$

(i.e., $p_{F}[X, Y]$ is the Hamiltonian vector field of the function $\sigma(X, Y)=$ $-\omega(X, Y))$.

Proof. Use Proposition 3.4 with $G=\{0\}$. Q.e.d.

Remark 3.4 By looking at the bidegree of the various terms in the decomposition of $d \omega$, we see that an $\mathcal{F}$-coupling form $\omega$ given by (52) is symplectic iff

$$
d^{\prime} \sigma=0, d^{\prime \prime} \sigma=-\partial \theta, d^{\prime} \theta=0, d^{\prime \prime} \theta=0
$$

Furthermore, if we give the pair $\left(H, \theta_{0,2}\right)$ where $d^{\prime} \theta=d^{\prime \prime} \theta=0, \partial \theta$ is a $d^{\prime \prime}$-closed $(2,1)$-form, and $[\partial \theta] \in H^{1}\left(M, \Phi^{2}\right)$, where $\Phi^{2}$ is the sheaf of germs of projectable cross sections of $\wedge^{2}$ (ann $\left.F\right)$, is a cohomological obstruction to the existence of a coupling form $\sigma_{2,0}$ associated with the given pair such that the corresponding form $\omega$ of (52) is symplectic. (See [12] for the cohomology space mentioned above.) 
Let $P^{\prime \prime}$ be a leaf-tangent bivector field on $(M, \mathcal{F})$. A transversally non degenerate extension of $P^{\prime \prime}$ is a bivector field $P$ such that $H=\sharp_{P}($ ann $F)$ is a normal bundle of the foliation $\mathcal{F}$ and the corresponding $(0,2)$-component of $P$ is the given bivector field $P^{\prime \prime}$. Then, of course, $P$ is $\mathcal{F}$-coupling. An example is given by

Proposition 3.5 If the foliation $F$ is a locally trivial fibration over a $2 q-$ dimensional basis $B$ and if $P^{\prime \prime}$ is a regular leaf-tangent Poisson structure on $(M, F)$, there exists a transversally non degenerate extension $P$ of $P^{\prime \prime}$ which satisfies condition (477).

Proof. By Weinstein's local structure theorem (e.g., [13]), $M$ may be covered by open, connected, neighborhoods endowed with local coordinates $\left(x^{a}, y^{\nu}, p_{\mu}, q^{\mu}\right)$ such that $x^{a}=$ const. define $\mathcal{F}, x^{a}=$ const., $y^{\nu}=$ const. define the symplectic foliation $\mathcal{S}$ of $P^{\prime \prime}$, and

$$
P^{\prime \prime}=\sum_{\mu} \frac{\partial}{\partial p_{\mu}} \wedge \frac{\partial}{\partial q^{\mu}} .
$$

Then,

$$
\sum_{a=1}^{q} \frac{\partial}{\partial x^{a}} \wedge \frac{\partial}{\partial x^{a+q}}+\sum_{\mu} \frac{\partial}{\partial p_{\mu}} \wedge \frac{\partial}{\partial q^{\mu}}
$$

are local transversally non degenerate extensions of the required type. They can be glued up to a global extension, which satisfies (47), by the pull-backs to $M$ of a partition of unity of the basis $B$ of the fibration $\mathcal{F}$. Q.e.d.

In [3, the process started by Proposition 3.5 is continued up to the construction of a coupling symplectic structure in the case where $P^{\prime \prime}$ is leaftangent symplectic and the fibers are connected, simply connected and compact. But, the difficulty to get transversally non degenerate, Poisson extensions of more general, leaf-tangent, Poisson structures $P$ growth if the rank of $P$ is lower. In particular, let us ask whether the structure $P^{\prime \prime}=0$ can be extended to a coupling Poisson structure $P$. For $P^{\prime \prime}=0$, (47) is satisfied, and (46) shows that the normal bundle $H$ must be integrable. Therefore extensions may exist only on a locally product manifold with structural foliations $\mathcal{F}, \mathcal{H}$, and, in view of (45), an extension is determined by a smooth family $\sigma$ of symplectic structures of the leaves of $\mathcal{H}$.

It is natural to inquire about the existence of a coupling Poisson structure (44) that is projectable onto the space of leaves of the given foliation $\mathcal{F}$. 
Projectability holds iff $\left(L_{Y} P^{\prime}\right)(\alpha, \beta)=0, \forall Y \in \Gamma F, \forall \alpha, \beta \in \Gamma($ ann $F)$, where, in fact, it is enough to take projectable 1 -forms $\alpha, \beta$. The answer is given by

Proposition 3.6 $P$ given by (44) is a projectable coupling Poisson bivector field iff i) $P^{\prime \prime}$ is Poisson, ii) $H$ is integrable; iii) the mod. F equivalent 2 -form $\sigma$ of $P^{\prime}$ is a transversal symplectic form of $\mathcal{F}$; iv) (47) holds.

Proof. If $P^{\prime}$ is projectable, so is the equivalent 2 -form $\sigma$, and we see that (45) implies iii) and that (46) implies the integrability of $H$. Conversely, iii), iv) imply (45), (47) and the projectability of $\sigma$ and $P^{\prime}$, which, together with ii), shows that the two sides of (46) are zero. Q.e.d.

Thus, projectable, $\mathcal{F}$-coupling Poisson tensors exist only on locally product manifolds $M$ with structural foliations $\mathcal{F}, \mathcal{H}$ where $\mathcal{H}$ is a leafwise symplectic foliation.

Remark 3.5 If an almost coupling, projectable, bivector field $P$, given by (44) is Poisson, $P^{\prime}$ alone necessarily is a Poisson bivector field, since by (15) the first two conditions (43) imply $\left[P^{\prime}, P^{\prime}\right]=0$. In the terminology of [15], $P^{\prime}$ yields a tame Hamiltonian structure of the foliation $\mathcal{F}$. Conversely, if we have such a tame structure with a corresponding Poisson structure $P^{\prime}$ of $M$, and a leaf-tangent Poisson structure $P^{\prime \prime}$, and if the third condition (43) holds, we get a projectable, almost coupling, Poisson structure on $(M, \mathcal{F})$. Of course, we may always take $P^{\prime \prime}=0$, hence, the tame Hamiltonian structures of $\mathcal{F}$ and the projectable, almost coupling, Poisson structures of $(M, \mathcal{F})$ are equivalent objects (not in a one-to-one correspondence, however).

\section{Vorobiev-Poisson structures}

In [16, Vorobiev extends an earlier construction of Montgomery-MarsdenRaţiu [10] to a large class of bundles of Lie coalgebras and obtains coupling Poisson structures in a neighborhood of the zero section of these bundles. Here, we give a simpler presentation of Vorobiev's results.

Let $p: \mathbb{G}^{*} \rightarrow B$ be a bundle of Lie coalgebras as in Example 2.3 with the supplementary conditions: i) $B$ is a symplectic manifold with the symplectic form $\omega$; ii) the dual Lie algebras bundle $\mathbb{G} \rightarrow B$ is the kernel of the (surjective) anchor $\rho: A \rightarrow T B$ of a transitive Lie algebroid $p: A \rightarrow B$. (We refer the reader to [7] for the general theory of Lie algebroids.) 
Let

$$
A=Q \oplus \mathbb{G}
$$

be a splitting of the vector bundle $A$, and $p_{Q}, p_{\mathbb{G}}$ the corresponding natural projections. Then $\left.\rho\right|_{Q}: Q \rightarrow T B$ is an isomorphism and we denote by $\gamma: T B \rightarrow Q$ its inverse. Obviously, we have

$$
\gamma(\rho(s))=p_{Q}(s), \quad \forall s \in A .
$$

Since we will make some local coordinate checks later, we fix the following notation now. Let $U \subseteq B$ be an open local-trivialization neighborhood of all the vector bundles above and $\left(x^{i}\right)(i=1, \ldots, n=\operatorname{dim} B)$ local coordinates on $U$. Then, we get the local basis $\mathbf{q}_{i}=\gamma\left(\partial / \partial x^{i}\right)$ of $Q$, and we may complete it by a local basis $\left(\mathbf{g}_{a}\right)$ of $\mathbb{G}(a=1, \ldots, k=\operatorname{rank} \mathbb{G})$ to a local basis of $A$. We will denote by $\left(\theta^{a}\right)$ the dual basis of $\left(\mathbf{g}_{a}\right)$ for the vector bundle $\mathbb{G}^{*}$. These bases define fiberwise local coordinates $\left(y^{a}\right)$ and $\left(y_{a}\right)$ on $\mathbb{G}, \mathbb{G}^{*}$, respectively. Since $\mathbb{G}=\operatorname{ker} \rho$ and $\rho$ is a morphism of Lie algebroids, the local expressions of the Lie bracket of $A$ must be of the form

$$
\begin{aligned}
& {\left[\mathbf{g}_{a}, \mathbf{g}_{b}\right]_{A}=\alpha_{a b}^{c}(x) \mathbf{g}_{c}, \quad\left[\mathbf{g}_{a}, \mathbf{q}_{i}\right]_{A}=\beta_{a i}^{c}(x) \mathbf{g}_{c},} \\
& {\left[\mathbf{q}_{i}, \mathbf{q}_{j}\right]_{A}=\gamma_{i j}^{c}(x) \mathbf{g}_{c}+\gamma_{i j}^{h}(x) \mathbf{q}_{h} .}
\end{aligned}
$$

(Again, we use the Einstein summation convention.) Finally, we indicate that a free use of the following natural identifications will be made below:

$$
\eta^{a} \frac{\partial}{\partial y^{a}} \Leftrightarrow \eta^{a} \mathbf{g}_{a}, \quad \mu_{a} \frac{\partial}{\partial y_{a}} \Leftrightarrow \mu_{a} \theta_{a} .
$$

Now, again since $\mathbb{G}=\operatorname{ker} \rho$, the formula

$$
\nabla_{X} \eta=[\gamma(X), \eta]_{A}, \quad X \in \mathcal{V}^{1}(B), \eta \in \Gamma \mathbb{G}
$$

defines a connection of the vector bundle $\mathbb{G}$. In the usual way, $\nabla$ yields a dual connection on $\mathbb{G}^{*}$ and connections on all the associated tensor bundles of $\mathbb{G}$, which we still denote by $\nabla$, except for situations where we want to emphasize the connection on $\mathbb{G}^{*}$ and, then, we will denote it by $\nabla^{*}$. Using (57), we see that the local components of $\nabla$ are given by

$$
\nabla_{\frac{\partial}{\partial x^{i}}} \mathbf{g}_{a}=\Gamma_{a i}^{b} \mathbf{g}_{b}, \quad \Gamma_{a i}^{b}=-\beta_{a i}^{b} .
$$


The Jacobi identity of the $A$-bracket is equivalent with

$$
\nabla C=0
$$

for the tensor $C$ defined by (33). Furthermore, notice that

$$
\rho\left([\gamma(X), \gamma(Y)]_{A}\right)=[X, Y]
$$

implies

$$
p_{Q}[\gamma(X), \gamma(Y)]_{A}=\gamma([X, Y]) .
$$

Then, by a straightforward computation, we see that the curvature $R_{\nabla}$ satisfies the condition

$$
R_{\nabla}(X, Y) \eta=\left[p_{\mathbb{G}}[\gamma(X), \gamma(Y)]_{A}, \eta\right]_{A} .
$$

Finally, we write

$$
T \mathbb{G}^{*}=\mathcal{H} \oplus \mathcal{V},
$$

with the projections $p_{\mathcal{H}}, p_{\mathcal{V}}$, where $\mathcal{V}$ is tangent to the fibers and $\mathcal{H}$ is the horizontal distribution of $\nabla^{*}$.

On $\mathbb{G}^{*}$, we have a triple $\left(P^{\prime \prime}, H, \sigma\right)$ as in Proposition 3.2 where $P^{\prime \prime}=\mathbb{L}$, $\mathbb{L}$ being the leaf-tangent Poisson bivector field defined by (32), $H=\mathcal{H}$ and

$$
\sigma_{z}(\mathcal{X}, \mathcal{Y})=\omega_{p(z)}(X, Y)-z\left(p_{\mathbb{G}}[\gamma(X), \gamma(Y)]_{A}\right),
$$

where $z \in \mathbb{G}^{*}, \mathcal{X}, \mathcal{Y} \in \Gamma \mathcal{H}$ are the horizontal lifts of $X=p_{*} \mathcal{X}, Y=p_{*} \mathcal{Y}$. The definition of $\sigma$ is completed by asking it to have bidegree $(2,0)$ with respect to 631).

The local expressions of the distribution $\mathcal{H}$ and the form $\sigma$ follow from well known formulas of differential geometry, and they are:

$$
\begin{gathered}
\mathcal{H}=\operatorname{span}\left\{\mathcal{X}_{i}=\frac{\partial}{\partial x^{i}}+\Gamma_{a i}^{b} y_{b} \frac{\partial}{\partial y_{a}}\right\}, \\
\sigma_{z}\left(\mathcal{X}_{i}, \mathcal{X}_{j}\right)=\omega_{i j}(x)-\gamma_{i j}^{c} y_{c},
\end{gathered}
$$

where $\mathcal{X}_{i}$ is the horizontal lift of $\partial / \partial x^{i}$, the coefficients $\gamma, \Gamma$ are those of (157) and (60), and $\omega_{i j}$ are the natural local components of $\omega$ on $B$. (Notice from (65) that the horizontal lifts of vector fields of $B$ are projectable with respect to the vertical foliation $\mathcal{V}$ of $\mathbb{G}^{*}$.) 
Proposition 4.1 [16] There exists a neighborhood $\mathcal{U}$ of $B$, seen as the zero section of $\mathbb{G}^{*}$, where the triple $(\mathbb{L}, \mathcal{H}, \sigma)$ defines a coupling Poisson bivector field.

Proof. Using (65), one gets

$$
\mathcal{X}(<z, \eta>)=<z, \nabla_{X} \eta>\quad\left(z \in \mathbb{G}^{*}, \eta \in \mathbb{G}, p(z)=p(\eta)\right) .
$$

Accordingly,

$$
\begin{gathered}
d \sigma(\mathcal{X}, \mathcal{Y}, \mathcal{Z})=\sum_{\operatorname{Cycl}(\mathcal{X}, \mathcal{Y}, \mathcal{Z})}\{\mathcal{X} \sigma(\mathcal{Y}, \mathcal{Z})+\sigma(\mathcal{X},[\mathcal{Y}, \mathcal{Z}])\} \\
=d \omega(X, Y, Z)-\sum_{\operatorname{Cycl}(X, Y, Z)}<z, \nabla_{X}\left(p_{\mathbb{G}}[\gamma(Y), \gamma(Z)]_{A}\right)> \\
\left.-\sum_{\operatorname{Cycl}(X, Y, Z)}<z, p_{\mathbb{G}}\left[\gamma(X), p_{Q}[\gamma(Y), \gamma(Z)]_{A}\right]_{A}\right)> \\
\left.=d \omega(X, Y, Z)-\sum_{\operatorname{Cycl}(X, Y, Z)}<z, p_{\mathbb{G}}\left[\gamma(X),[\gamma(Y), \gamma(Z)]_{A}\right)\right]_{A}>=0,
\end{gathered}
$$

and, in view of the closedness of $\omega$ and of the Jacobi identity for $A$, (45) holds.

Furthermore, we have

$$
\left(L_{\mathcal{X}} \mathbb{L}\right)_{z}(\eta, \nu)=\mathcal{X}\left(<z, C(\eta, \nu>)-\mathbb{L}_{z}\left(L_{\mathcal{X}} \eta, \nu\right)-\mathbb{L}_{z}\left(\eta, L_{\mathcal{X}} \nu\right),\right.
$$

where $\eta, \nu \in \Gamma T^{*} \mathbb{G}^{*} \Leftrightarrow \Gamma \mathbb{G}$. The Lie derivatives in the right hand side may be evaluated at $z \in \mathbb{G}^{*}$ by using the identification $z \Leftrightarrow y_{a}\left(\partial / \partial y_{a}\right)$, and formulas (65), (67):

$$
<L_{\mathcal{X}} \eta, y_{a} \frac{\partial}{\partial y_{a}}>=\mathcal{X}<z, \eta>-<\eta,\left[\mathcal{X}, y_{a} \frac{\partial}{\partial y_{a}}\right]>=<z, \nabla_{X} \eta>.
$$

As a consequence, we obtain

$$
\left(L_{\mathcal{X}} \mathbb{L}\right)_{z}(\eta, \nu)=<z,\left(\nabla_{X} C\right)(\eta, \nu)>,
$$

and (47) follows from (61).

Finally, (46) can be checked as follows. From (31), (33), and (62) we get

$$
\mathbb{L}_{z}\left(p_{\mathbb{G}}[\gamma(X), \gamma(Y)]_{A}, \eta\right)=<z,\left[p_{\mathbb{G}}[\gamma(X), \gamma(Y)]_{A}, \eta\right]_{A}>
$$




$$
=<z, R_{\nabla}(X, Y) \eta>=-<R_{\nabla^{*}}(X, Y) z, \eta>,
$$

where again $z \Leftrightarrow y_{a}\left(\partial / \partial y_{a}\right)$. Equivalently,

$$
\sharp_{\mathbb{L}_{z}}\left(p_{\mathbb{G}}[\gamma(X), \gamma(Y)]_{A}\right)=-R_{\nabla^{*}}(X, Y) z .
$$

But, by classical connection theory, $-R_{\nabla^{*}}(X, Y) z$ is equal to $p_{\mathcal{V}}[\mathcal{X}, \mathcal{Y}](z)$, here identified with a vector of the fiber $\mathbb{G}_{p(z)}$. On the other hand, $\forall \mu \in \Gamma \mathbb{G}$ the $(0,1)$-component of $\left.d<z, \mu_{z}\right\rangle$ with respect to (63) identifies with $\mu$. Accordingly, (64) yields

$$
\sharp_{\mathbb{L}_{z}}\{d[\sigma(\mathcal{X}, \mathcal{Y})]\}=P_{\mathcal{V}}[\mathcal{X}, \mathcal{Y}],
$$

which is (46) in our case.

Therefore, since $\sigma$ is non degenerate on a neighborhood $\mathcal{U}$ of the zero section of $\mathbb{G}^{*}$, there exists a corresponding coupling Poisson structure on $\mathcal{U}$. Q.e.d.

Definition 4.1 The coupling Poisson structure defined by Proposition 4.1 on the neighborhood $\mathcal{U}$ of $B$ will be called a Vorobiev-Poisson structure.

Remark 4.1 [16] If the distribution

$$
\mathcal{D}=\left\{X \in T B / p_{\mathbb{G}}[\gamma(X), \gamma(Y)]_{A}=0, \forall Y \in T B\right\}
$$

is coisotropic with respect to $\omega$, the Vorobiev-Poisson structure is globally coupling (i.e., $\mathcal{U}=\mathbb{G}^{*}$ ). Indeed, the local equations of $\mathcal{D}$ are

$$
\gamma_{i j}^{a} X^{i}=0 \quad\left(X=X^{i} \frac{\partial}{\partial x^{i}}\right),
$$

and $\mathcal{D}$ is coisotropic iff the equations (69) together with $\omega_{i j} X^{i} Y^{j}=0$ imply $\gamma_{i j}^{a} Y^{i}=0$. Now, assume that $\mathcal{X}=X^{i} \mathcal{X}_{i} \in$ ann $\sigma_{z}$ i.e.,

$$
\left(\omega_{i j}-y_{c} \gamma_{i j}^{c}\right) X^{i} Y^{j}=0, \quad \forall \mathcal{Y}=Y^{j} \mathcal{X}_{j} .
$$

Then, we get $\omega_{i j} X^{i} Y^{j}=0$ for all the vectors $Y \in \mathcal{D}_{p(z)}$ and $X=X^{i}\left(\partial / \partial x^{i}\right)$ must belong to $\mathcal{D}$. This reduces (70) to $X \in \operatorname{ann} \omega_{p(z)}$ and, since $\omega$ is non degenerate, we get $X=0$ and $\mathcal{X}=0$, i.e., $\sigma_{z}$ is non degenerate.

Furthermore, one has the following important result. 
Proposition 4.2 [16] The Vorobiev-Poisson structures defined by two splittings

$$
A=Q \oplus \mathbb{G}, \quad A=\tilde{Q} \oplus \mathbb{G}
$$

on neighborhoods $\mathcal{U}_{1}, \mathcal{U}_{2}$ of $B$ in $\mathbb{G}^{*}$ are Poisson-equivalent in a neighborhood $V \subseteq \mathcal{U}_{1} \cap \mathcal{U}_{2}$

Proof. The notation below is that of Proposition 4.1 with the addition of a tilde for everything related to the second splitting .

The difference $\phi=\tilde{\gamma}-\gamma$ is a $\mathbb{G}$-valued 1-form on $B$ and, if $s \in \Gamma A$ has the two decompositions

$$
s=p_{\mathbb{G}}(s)+p_{Q}(s)=\tilde{p}_{\mathbb{G}}(s)+p_{\tilde{Q}}(s),
$$

(56) implies

$$
\tilde{p}_{\mathbb{G}}(s)=p_{\mathbb{G}}(s)-\phi(\rho(s)), \quad p_{\tilde{Q}}(s)=p_{Q}(s)+\phi(\rho(s)) .
$$

Furthermore, on $\mathbb{G}^{*}$ we get a scalar 1 -form $\psi \in$ ann $\mathcal{V}$ by means of the formula

$$
\psi_{z}(\mathcal{X})=<z, \phi(X)>, \quad z \in \mathbb{G}^{*} .
$$

Using $\phi$, we may define a homotopy, i.e., a family of splittings $A=Q_{t} \oplus \mathbb{G}$, given by the projectors

$$
p_{Q_{t}}=p_{Q}+t(\phi \circ \rho), \quad t \in \mathbb{R},
$$

which is such that $Q_{0}=Q, Q_{1}=\tilde{Q}$, with the corresponding homotopy class $P_{t}$ of Vorobiev-Poisson bivector fields defined by the triples $\left(\mathbb{L}, \mathcal{H}_{t}, \sigma_{t}\right)$ of the connection $\nabla^{t}$ associated to (74) via (59).

With (60) and the definition of $\phi$, it is easy to compute the connection coefficients of $\nabla^{t}$ and get

$$
\mathcal{H}_{t}=\operatorname{span}\left\{\mathcal{X}_{t, i}=\mathcal{X}_{i}+t \alpha_{a c}^{b} \phi_{i}^{c} y_{b} \frac{\partial}{\partial y_{a}}\right\},
$$

where the components $\phi_{i}^{c}$ are given by

$$
\phi\left(\frac{\partial}{\partial x^{i}}\right)=\phi_{i}^{c} \mathbf{g}_{c} .
$$


The corresponding basis of ann $\mathcal{H}_{t}=\mathcal{V}^{*}$ consists of the forms

$$
\mu_{t, a}=\mu_{a}-t \alpha_{a c}^{b} \phi_{i}^{c} y_{b} d x^{i}
$$

where $\mu_{a}=\mu_{0, a}$. From (75) we get the horizontal lift of $X \in T B$ to $\mathcal{H}_{t}$

$$
\mathcal{X}_{t}(z)=\mathcal{X}(z)-t \operatorname{coad}_{\phi(X)}(\mathbb{E}(z)), \quad z \in \mathbb{G}^{*}, \mathbb{E}(z)=y_{a} \frac{\partial}{\partial y_{a}} \Leftrightarrow y_{a} \theta^{a}
$$

$\left(\mathbb{E}\right.$ is the infinitesimal homothety of $\left.\mathbb{G}^{*}\right)$. Notice also that (177) may be seen as a bijection $\lambda \mapsto \lambda_{t}$ between ann $\mathcal{H}$ and ann $\mathcal{H}_{t}$ given by:

$$
\lambda_{t}=\lambda-t L_{\sharp_{\mathbb{L}} \lambda} \psi
$$

where $\psi$ is the 1 -form (73)).

Now, we can compute the 2 -form $\sigma_{t}$ of the triple $\left(\mathbb{L}, \mathcal{H}_{t}, \sigma_{t}\right)$. Since the horizontal lifts are such that the differences $\tilde{\mathcal{X}}-\mathcal{X}, \tilde{\mathcal{Y}}-\mathcal{Y}$ are vertical, and using (72), we get

$$
\sigma_{t}(z)\left(\mathcal{X}_{t}, \mathcal{Y}_{t}\right)=\sigma_{t}(z)(\mathcal{X}, \mathcal{Y})=\sigma(z)(\mathcal{X}, \mathcal{Y})
$$

$-<z, t[\gamma(X), \phi(Y)]_{A}-t[\gamma(Y), \phi(X)]_{A}-t \phi([X, Y])+t^{2}[\phi(X), \phi(Y)]_{A}>$.

Furthermore, using (67), (80) becomes

$$
\sigma_{t}\left(\mathcal{X}_{t}, \mathcal{Y}_{t}\right)=\sigma(\mathcal{X}, \mathcal{Y})-t d \psi(\mathcal{X}, \mathcal{Y})-t^{2} \mathbb{L}(\phi(X), \phi(Y))
$$

As in [16], at this point we define a time-dependent vector field $\Xi_{t} \in \Gamma\left(\mathcal{H}_{t}\right)$ by

$$
\Xi_{t}=\sharp_{P_{t}} \psi \text {. }
$$

Equivalently, and with the notation of (44), we have

$$
b_{\sigma_{t}} \Xi_{t}=i\left(\Xi_{t}\right) \sigma_{t}=-\psi \text {. }
$$

The vector field $\Xi_{t}$ is equivalent with the autonomous vector field $\tilde{\Xi}=\Xi_{t}+$ $\partial / \partial t$ on $\mathbb{G}^{*} \times \mathbb{R}$, and we will prove that the flow $\Phi_{t}$ of $\tilde{\Xi}$ preserves the lift of the tensor field $P_{t}$ to $\mathbb{G}^{*} \times \mathbb{R}$. Then the projection of the diffeomorphism $\Phi_{1}$ onto $\mathbb{G}^{*}$ will be the required equivalence of coupling Poisson structures.

Thus, the proof will be accomplished if we show that

$$
L_{\tilde{\Xi}} P_{t}=0 .
$$


Condition (84) obviously holds if one of the arguments is $d t$. In the other cases, we shall see that (84) is a consequence of the fact that $P_{t}$ satisfies the characteristic conditions (43) of the coupling Poisson tensors for all $t$.

Indeed, the third condition (43) for $\alpha=\psi$ yields

$$
\left(L_{\Xi_{t}} P_{t}\right)\left(\lambda_{t}, \mu_{t}\right)=0
$$

and (79) yields

$$
L_{\frac{\partial}{\partial t}} P_{t}\left(\lambda_{t}, \mu_{t}\right)=\frac{\partial}{\partial t}(\mathbb{L}(\lambda, \mu)=0 .
$$

Therefore (84) holds for arguments in ann $\mathcal{H}_{t}$.

For mixed arguments $\alpha \in$ ann $\mathcal{V}, \lambda_{t} \in$ ann $\mathcal{H}_{t}$ we get

$$
\left(L_{\Xi_{t}} P_{t}\right)\left(\alpha, \lambda_{t}\right)=\alpha\left(\left[\sharp_{P_{t}^{\prime \prime}} \lambda_{t}, \sharp_{P_{t}^{\prime}} \psi\right]\right)-\lambda_{t}\left(\left[\sharp_{P_{t}^{\prime}} \alpha, \sharp_{P_{t}^{\prime}} \psi\right]\right)
$$

and, if the second term is replaced in agreement with the second condition (43) and $\lambda_{t}$ is expressed by (179), the result is

$$
\left(L_{\Xi_{t}} P_{t}\right)\left(\alpha, \lambda_{t}\right)=P_{t}^{\prime}\left(L_{\sharp_{P_{t}^{\prime \prime}}} \lambda_{t} \psi, \alpha\right)=P_{t}^{\prime}\left(L_{\sharp_{\mathbb{L}} \lambda} \psi, \alpha\right) .
$$

On the other hand, using (179) again, we get

$$
\left(L_{\frac{\partial}{\partial t}} P_{t}\right)\left(\alpha, \lambda_{t}\right)=-P_{t}\left(\alpha, \frac{\partial \lambda_{t}}{\partial t}\right)=P_{t}^{\prime}\left(\alpha, L_{\sharp \mathbb{L} \lambda} \psi\right) .
$$

Therefore, (84) holds for mixed arguments.

Finally, for $\alpha, \beta \in$ ann $\mathcal{V}$, and if $\mathcal{X}_{t}=\sharp_{P_{t}^{\prime}} \alpha, \mathcal{Y}_{t}=\sharp_{P_{t}^{\prime}} \beta$, the first condition (43)) for $\gamma=\psi$ yields

$$
\left(L_{\Xi_{t}} P_{t}\right)(\alpha, \beta)=d \psi\left(\mathcal{X}_{t}, \mathcal{Y}_{t}\right)
$$

Furthermore, with (75), we deduce

$$
\left(L_{\Xi_{t}} P_{t}\right)(\alpha, \beta)=d \psi(\mathcal{X}, \mathcal{Y})+2 t \mathbb{L}(\phi(X), \phi(Y))
$$

where the notation is that of the formulas (78), (81). Then,

$$
\begin{gathered}
\left(L_{\partial \partial t} P_{t}\right)(\alpha, \beta)=\frac{\partial}{\partial t}\left[P_{t}(\alpha, \beta)\right]=\frac{\partial}{\partial t}\left[\sigma_{t}\left(\mathcal{X}_{t}, \mathcal{Y}_{t}\right)\right] \\
=-d \psi(\mathcal{X}, \mathcal{Y})-2 t \mathbb{L}(\phi(X), \phi(Y)) .
\end{gathered}
$$

Therefore, (84) also holds for two arguments in ann $\mathcal{V}$. Q.e.d. 
Remark 4.2 It is important to notice that the equivalence of Poisson structures given by Proposition 4.2 does not preserve the foliation $\mathcal{V}$. On the other hand, we also notice that the triple $(-\mathbb{L}, H, \tilde{\sigma})$, where

$$
\tilde{\sigma}_{z}(\mathcal{X}, \mathcal{Y})=\omega_{p(z)}(X, Y)+z\left(p_{\mathbb{G}}[\gamma(X), \gamma(Y)]_{A}\right)
$$

is also a coupling Poisson structure on a neighborhood of $B$ in $\mathbb{G}^{*}$. This follows from Remark 3.2 since $-\tilde{\sigma}=\sigma-2 p^{*} \omega$. The new structure also satisfies the equivalence property of Proposition 4.2. The proof is the same except for a change of sign in the definition of the vector field $\Xi_{t}$. This structure will also be called a Vorobiev-Poisson structure.

Following [16, it is possible to apply the previous construction to an embedded leaf $S$ of a Poisson manifold $(M, P)$. The Lie algebroid structure of the cotangent bundle $T^{*} M$ restricts to a transitive Lie algebroid $\left.T^{*} M\right|_{S} \longrightarrow$ $S$ and the kernel of the anchor of this algebroid is the conormal bundle of $S$, i.e., the annihilator of $T S$ in $\left.T^{*} M\right|_{S}$. Let $N S$ be a normal bundle of $S$ (i.e., $\left.T M\right|_{S}=T S \oplus N S$ ) and $U$ a tubular neighborhood of $S$ with the fibers tangent to $N S$. At the points of $S$ there exist local adapted coordinates $\left(x^{\alpha}, x^{\kappa}\right)(\alpha=1, \ldots, \operatorname{codim}(S) ; \kappa=\operatorname{codim}(S)+1, \ldots, \operatorname{dim}(M))$ such that the local equations of $S$ are $x^{\alpha}=0$ and

$$
N^{*} S=\operatorname{ann}(T S)=\operatorname{span}\left\{\left.d x^{\alpha}\right|_{S}\right\}, \quad T^{*} S=\operatorname{ann}(N S)=\operatorname{span}\left\{\left.d x^{\kappa}\right|_{S}\right\} .
$$

The vector bundles $N^{*} S$ and $T^{*} S$ may play the role of $\mathbb{G}$ and $Q$ of Vorobiev's construction, respectively, and the bases (86) may have the role of the bases $\left(\mathbf{g}_{a}\right),\left(\mathbf{q}_{i}\right)$ of (157). If $P^{\alpha \beta}, P^{\alpha \kappa}, P^{\kappa \nu}$ are the local components of $P$ with respect to the local coordinates defined above, one has $\left.P^{\alpha \beta}\right|_{S}=\left.P^{\alpha \nu}\right|_{S}=0$, whence

$$
\left.\frac{\partial P^{\alpha \beta}}{\partial x^{\kappa}}\right|_{S}=0,\left.\quad \frac{\partial P^{\alpha \nu}}{\partial x^{\kappa}}\right|_{S}=0 .
$$

Accordingly, the brackets (57) of the present case will be

$$
\begin{aligned}
& \left\{\left.d x^{\alpha}\right|_{S},\left.d x^{\beta}\right|_{S}\right\}=\left.\left.\frac{\partial P^{\alpha \beta}}{\partial x^{\gamma}}\right|_{S} d x^{\gamma}\right|_{S},\left\{\left.d x^{\alpha}\right|_{S},\left.d x^{\kappa}\right|_{S}\right\}=\left.\left.\frac{\partial P^{\alpha \kappa}}{\partial x^{\gamma}}\right|_{S} d x^{\gamma}\right|_{S}, \\
& \left\{\left.d x^{\kappa}\right|_{S},\left.d x^{\nu}\right|_{S}\right\}=\left.\left.\frac{\partial P^{\kappa \nu}}{\partial x^{\gamma}}\right|_{S} d x^{\gamma}\right|_{S}+\left.\left.\frac{\partial P^{\kappa \nu}}{\partial x^{\theta}}\right|_{S} d x^{\theta}\right|_{S} .
\end{aligned}
$$

(In the formulas above, $\beta, \gamma$ have the same domain as $\alpha$ and $\nu, \theta$ have the same domain as $\kappa$.) 
Therefore, one has a Vorobiev-Poisson structure on a neighborhood of $S$ and the local formulas (31), (65) and (66) show that the associated triple is given by

$$
\begin{aligned}
& P^{\prime \prime}=\left.\frac{1}{2} \xi^{\gamma} \frac{\partial P^{\alpha \beta}}{\partial x^{\gamma}}\right|_{x^{\gamma}=0} \frac{\partial}{\partial \xi^{\alpha}} \wedge \frac{\partial}{\partial \xi^{\beta}}, \\
& H=\operatorname{span}\left\{\frac{\partial}{\partial x^{\kappa}}-\left.\xi^{\gamma} \frac{\partial P^{\alpha \kappa}}{\partial x^{\gamma}}\right|_{x^{\gamma}=0} \frac{\partial}{\partial \xi^{\alpha}}\right\}, \\
& \sigma=\frac{1}{2}\left(p_{\kappa \nu}-\left.\xi^{\gamma} \frac{\partial P^{\kappa \nu}}{\partial x^{\gamma}}\right|_{x^{\gamma}=0}\right) d x^{\kappa} \wedge d x^{\nu},
\end{aligned}
$$

where $\xi^{\alpha}$ are fiber coordinates in the normal bundle $N S, p_{\kappa \nu} P^{\nu \theta}=\delta_{\kappa}^{\theta}$ and the indices take the same values as in (87). The invariant expression of the 2 -form $\sigma$ of $(88)$ is

$$
\sigma_{Z}(\mathcal{X}, \mathcal{Y})=\omega_{p(Z)}(X, Y)-<Z,\{\xi, \eta\}_{P}>
$$

where $Z \in N S, \xi, \eta \in T^{*} S, X=\sharp_{P} \xi, Y=\sharp_{P} \eta$ and the bracket of 1-forms is that given by (5).

The Vorobiev-Poisson structure (88) is defined up to Poisson equivalence and its $S$-transversal part may be seen as a linear approximation of the $S$ transversal part of the original Poisson structure $P$.

\section{$5 \quad$ Jacobi structures on foliated manifolds}

In this section we discuss similar problems for Jacobi structures. We recall that a Jacobi structure is a Lie bracket of the local type on the algebra of differentiable functions on a manifold, $C^{\infty}(M)$. Such a bracket must be of the form

$$
\{f, g\}=\Lambda(d f, d g)+f(E g)-g(E f) \quad\left(f, g \in C^{\infty}(M)\right)
$$

where $\Lambda \in \mathcal{V}^{2}(M), E \in \mathcal{V}^{1}(M)$ satisfy the conditions

$$
[\Lambda, \Lambda]=2 E \wedge \Lambda, \quad L_{E} \Lambda=0 .
$$

Accordingly, we refer to such a pair $(\Lambda, E)$ as a Jacobi structure. For a Jacobi structure, $i m \sharp_{\Lambda}+\operatorname{span}\{E\}$ is an integrable generalized distribution, called the characteristic distribution, with either locally conformal symplectic or contact leaves. As general references on Jacobi structures, we quote [2] and 14. 
We also recall the fundamental fact that the Jacobi structure $(\Lambda, E)$ on the manifold $M$ is equivalent with the homogeneous Poisson structure

$$
P=e^{-t}\left(\Lambda+\frac{\partial}{\partial t} \wedge E\right), \quad t \in \mathbb{R}
$$

on $M \times \mathbb{R}$ [2, 14].

The general conditions for a couple $(\Lambda, E)$ to define a Jacobi structure on the foliated manifold $(M, \mathcal{F})$ with a normal bundle $H$ of $\mathcal{F}$ may be written down using (15)-(20) to express (91).

Definition 5.1 A pair $(\Lambda, E)$ that consists of a bivector field $\Lambda$ and a vector field $E$ on a manifold $M$ is pre-coupling with respect to a foliation $\mathcal{F}$ if $\sharp_{\Lambda}($ ann $F) \cap F=\{0\}(F=T \mathcal{F})$. If $E$ is tangent to $\mathcal{F}$ and $\Lambda \in \Gamma \wedge^{2} F$ the pair $(\Lambda, E)$ is said to be a leaf-tangent pair.

In the remaining part of the paper all the pairs $(\Lambda, E)$ on $(M, \mathcal{F})$ will be pre-coupling. In particular, a leaf-tangent pair obviously is pre-coupling.

Definition 5.21 ) The pre-coupling pair $(\Lambda, E)$ is of the $\mathcal{F}$-tangent or the $\mathcal{F}$-normal type if the vector field $E$ is always tangent, respectively, never tangent to $\mathcal{F}$. 2) $(\Lambda, E)$ is $\mathcal{F}$-almost coupling via $H$ if $\sharp_{\Lambda}($ ann $F) \subseteq H$ for the normal bundle $H$ of $\mathcal{F}$. 3) $(\Lambda, E)$ is $\mathcal{F}$-coupling of the first kind if it is of the tangent type and $\sharp_{\Lambda}($ ann $F)$ is a normal bundle $H$ of $\mathcal{F}$. 4) $(\Lambda, E)$ is $\mathcal{F}$-coupling of the second kind if $\sharp_{\Lambda}($ ann $F)$ is a normal bundle $H$ of $\mathcal{F}$ that contains $E$. 5) $(\Lambda, E)$ is $\mathcal{F}$-coupling of the third kind if it is of the normal type and $H^{\prime}=\sharp_{\Lambda}[\operatorname{ann}(F \oplus \operatorname{span}\{E\})]$ is a complementary distribution of $F \oplus(\operatorname{span}\{E\})$.

In the first and second coupling cases, we always take the normal bundle $H$ defined by the coupling condition and in the third case we always take $H=H^{\prime} \oplus \operatorname{span}\{E\}$. For any foliation $\mathcal{F}$ and any bivector field $\Lambda$ we have

$$
F \cap \sharp_{\Lambda}(\operatorname{ann} F)=\sharp_{\Lambda}\left(\left.\operatorname{ker} \Lambda\right|_{\text {ann } F}\right) .
$$

Hence, $\sharp_{\Lambda}($ ann $F)$ is normal to $\mathcal{F}$ iff

$$
\operatorname{rank}\left(\left.\Lambda\right|_{\text {ann } \mathcal{F}}\right)=\operatorname{codim} \mathcal{F} .
$$

Thus, if $E \in \Gamma F$, condition (93) is equivalent with coupling of the first kind while, on the contrary, if $E \in i m \sharp_{\Lambda}$, (93) is equivalent with second kind 
coupling. Similarly, we see that the third kind coupling condition holds iff $E$ is never tangent to $\mathcal{F}$ and

$$
\operatorname{rank}\left(\left.\Lambda\right|_{\operatorname{ann}(\mathcal{F} \oplus \operatorname{span}\{E\})}\right)=\operatorname{codim} \mathcal{F}-1
$$

Example 5.1 Assume that a characteristic leaf $L$ of the Jacobi manifold $(M, \Lambda, E)$ is transversal to the foliation $\mathcal{F}$. If $L$ is locally conformal symplectic, the condition that $E$ is not in $F$ and (93) hold along $L$ hence, by continuity, also hold in a neighborhood $U$ of $L$. Therefore, $(\Lambda, E)$ is $\mathcal{F}$ coupling of the second kind on $U$. If $L$ is a contact manifold, the conditions for $(\Lambda, E)$ to be coupling of the third kind hold along $L$ hence, also on a neighborhood $U$ of $L$. In particular, for any Jacobi structure, any embedded even-dimensional leaf $L$, has a tubular neighborhood $U$ where the Jacobi structure is coupling of the second kind with the fibers of the tubular structure and any embedded odd-dimensional leaf $L$ has a tubular neighborhood $U$ of $L$ where the Jacobi structure is coupling of the third kind with the fibers of the tubular structure.

Example 5.2 Let $\mathbb{G} \longrightarrow B$ be a bundle of Lie algebras, which is the kernel of a transitive Lie algebroid over a symplectic manifold, and assume that there exists a global cross section $\zeta$ of the dual bundle $\mathbb{G}^{*}$ that vanishes on the derived algebras of the fibers of $\mathbb{G}$. Then $\zeta$ may be seen as a vertical vector field on $\mathbb{G}^{*}$, and straightforward computations show that the pair

$$
\Lambda=\mathbb{L}+\mathbb{E} \wedge \zeta, \quad E=-\zeta,
$$

where $\mathbb{L}, \mathbb{E}$ are defined by (132), (178), is a leaf-tangent Jacobi structure on $\mathbb{G}^{*}$ with the vertical foliation $\mathcal{V}$ ). (This is a particular case of the general result of [4.) Furthermore, if $P$ is a Vorobiev-Poisson structure on a neighborhood of $B$ in $\mathbb{G}^{*}$ with the vertical part $\mathbb{L}$, the pair $\Lambda_{P}=P+\mathbb{E} \wedge \zeta, E=-\zeta$ is coupling of the first kind on the same neighborhood, which is not a Jacobi structure, however.

It is clear that coupling implies almost coupling, and coupling of either the second or the third kind implies the normal type condition. Coupling of the second kind may exist only if the codimension of $\mathcal{F}$ is even and coupling of the third kind may exist only if the codimension of $\mathcal{F}$ is odd. Obviously, 
condition (93) holds iff the bivector field $P$ defined on $M \times \mathbb{R}$ by (92) is coupling for the foliation $\mathcal{F} \times \mathbb{R}$. Therefore, this property of $P$ holds for $(\Lambda, E)$ coupling of the first or second kind, and the two situations are differentiated by $E \in \Gamma F, E \in \sharp_{\Lambda}($ ann $F)$, respectively. Finally, if $(\Lambda, E)$ is of the normal type, coupling of the third kind holds iff $P$ is coupling for the pullback of $\mathcal{F}$ to $M \times \mathbb{R}$ and, then, the corresponding normal bundle is $\tilde{H} \oplus \operatorname{span}\left\{\frac{\partial}{\partial t}\right\}$, where $\tilde{H}$ is the pullback of $\sharp_{\Lambda}[\operatorname{ann}(F \oplus \operatorname{span}\{E\})]$ to $M \times \mathbb{R}$.

Furthermore, we remark that a conformal change

$$
\{f, g\}^{a}=\frac{1}{a}\{a f, a g\} \quad\left(a=e^{b}, b \in C^{\infty}(M)\right)
$$

of an $\mathcal{F}$-coupling Jacobi structure of either the second or the third kind $\{f, g\}$ leads to a Jacobi structure of the same type. Indeed, if the original structure is given by $(\Lambda, E)$, the new structure is given by [2, 14]

$$
\Lambda^{a}=a \Lambda, E^{a}=a E+\sharp_{\Lambda}(d a) .
$$

If the original structure is coupling of the first kind, (97) is coupling of the first kind iff $a=$ const.

With the definitions above, we get

Proposition 5.1 On a foliated manifold $(M, \mathcal{F})$, a pair $(\Lambda, E)$ of the tangent type, which is almost coupling via a normal bundle $H$, is a Jacobi structure iff

$$
\Lambda=\Lambda_{2,0}^{\prime}+\Lambda_{0,2}^{\prime \prime}
$$

with respect to the bigrading defined by $H$ and

$$
\begin{gathered}
d^{\prime} \gamma\left(\sharp_{\Lambda^{\prime}} \alpha, \sharp_{\Lambda^{\prime}} \beta\right)-\left(L_{\sharp_{\Lambda^{\prime}} \gamma} \Lambda^{\prime}\right)(\alpha, \beta)=0, \\
\left(L_{\sharp_{\Lambda^{\prime \prime}} \lambda} \Lambda^{\prime}\right)(\alpha, \beta)+\lambda\left(\left[\sharp_{\Lambda^{\prime}} \alpha, \sharp_{\Lambda^{\prime}} \beta\right]\right)=\lambda(E) \Lambda^{\prime}(\alpha, \beta), \\
\left(L_{\sharp_{\Lambda^{\prime}} \alpha} \Lambda^{\prime \prime}\right)(\lambda, \mu)=0, \\
d^{\prime \prime} \nu\left(\sharp_{\Lambda^{\prime \prime}} \lambda, \sharp_{\Lambda^{\prime \prime}} \mu\right)-\left(L_{\sharp_{\Lambda^{\prime \prime}}} \Lambda^{\prime \prime}\right)(\lambda, \mu)=\left(E \wedge \Lambda^{\prime \prime}\right)(\lambda, \mu, \nu) \\
L_{E} \Lambda^{\prime \prime}=0, L_{E} \Lambda^{\prime}=0
\end{gathered}
$$

for 1 -forms $\alpha, \beta, \gamma$ of bidegree $(1,0)$ and $\lambda, \mu, \nu$ of bidegree $(0,1)$. 
Proof. From (15)-(201), and since $E \in \Gamma F$, we see that the first condition (91) is equivalent to (98). Then, by looking at $L_{E} \Lambda=0$ for arguments of bidegree $(1,0),(0,1)$ we get the first condition (100) first, and then the second. Q.e.d.

Together, the last condition (99) and the first condition (100) are equivalent with the fact that $\left(\Lambda^{\prime \prime}, E\right)$ is a leaf-tangent Jacobi structure on $(M, \mathcal{F})$, and a pair $\left(\Lambda_{0,2}, E_{0,1}\right)$ is a leaf-tangent Jacobi structure iff these two conditions hold.

Furthermore, if $\left(\Lambda=\Lambda^{\prime}+\Lambda^{\prime \prime}, E\right)$ is coupling of the first kind, we may use the $(2,0)$-form $\sigma$ that is equivalent, mod. $F$ with $\Lambda^{\prime}$, as we did in the Poisson case (see Proposition [3.2), and we get

Proposition 5.2 If the pair $(\Lambda, E)$ is coupling of the first kind, it is a Jacobi structure iff $\left(\Lambda^{\prime \prime}, E\right)$ is leaf-tangent Jacobi and

$$
\begin{gathered}
d^{\prime} \sigma=0, L_{X} \Lambda^{\prime \prime}=0, \\
-p_{F}[X, Y]=\sharp_{\Lambda^{\prime \prime}}\{d[\sigma(X, Y)]\}-\sigma(X, Y) E, \\
L_{E} \Lambda^{\prime}=0 .
\end{gathered}
$$

for all the vector fields $X, Y \in \mathcal{V}_{p r}^{1,0}(M)$.

Notice also that the last condition (101) is equivalent with

$$
E(\sigma(X, Y))=0,[E, X]=0 \quad X, Y \in \mathcal{V}_{p r}^{1,0}(M) .
$$

Similarly, we get

Proposition 5.3 An almost coupling pair $(\Lambda, E)$ of the normal type is a Jacobi structure iff (98) holds and the following conditions are satisfied:

$$
\begin{aligned}
& d^{\prime} \gamma\left(\sharp_{\Lambda^{\prime}} \alpha, \sharp_{\Lambda^{\prime}} \beta\right)-\left(L_{\sharp_{\Lambda^{\prime}}} \Lambda^{\prime}\right)(\alpha, \beta)=E \wedge \Lambda^{\prime}(\alpha, \beta, \gamma), \\
&\left(L_{\sharp_{\Lambda^{\prime \prime}}} \Lambda^{\prime}\right)(\alpha, \beta)+\lambda\left(\left[\sharp_{\Lambda^{\prime}} \alpha, \sharp_{\Lambda^{\prime}} \beta\right]\right)=0, \\
&\left(L_{\sharp_{\Lambda^{\prime}}} \Lambda^{\prime \prime}\right)(\lambda, \mu)=\alpha(E) \Lambda^{\prime \prime}(\lambda, \mu), \\
& d^{\prime \prime} \nu\left(\sharp_{\Lambda^{\prime \prime}} \lambda, \sharp_{\Lambda^{\prime \prime}} \mu\right)-\left(L_{\sharp_{\Lambda^{\prime \prime}}} \Lambda^{\prime \prime}\right)(\lambda, \mu)=0, \\
&\left(L_{E} \Lambda^{\prime}\right)(\alpha, \beta)=0,\left(L_{E} \Lambda^{\prime \prime}\right)(\lambda, \mu)=0,\left(L_{E} \Lambda^{\prime}\right)(\alpha, \lambda)=\left(L_{E} \Lambda^{\prime \prime}\right)(\lambda, \alpha) .
\end{aligned}
$$


In the situation of Proposition 5.3, the last condition (103) is equivalent with the fact that $\Lambda^{\prime \prime}$ is a leaf-tangent Poisson structure on $(M, \mathcal{F})$ and $E$ is an infinitesimal automorphism of the former. If $E$ is a projectable vector field conditions (104) become again (100). Furthermore, in the case of a coupling pair of the second kind, we may again use a $(2,0)$-form $\sigma$ mod. $F$-equivalent with $\Lambda^{\prime}$, and get

Proposition 5.4 If the pair $(\Lambda, E)$ is $\mathcal{F}$-coupling of the second kind, it is a Jacobi structure iff $\Lambda^{\prime \prime}$ is a leaf-tangent Poisson structure, (104) hold and

$$
d^{\prime} \sigma=\left(b_{\sigma} E\right) \wedge \sigma, L_{X} \Lambda^{\prime \prime}=\sigma(X, E) \Lambda^{\prime \prime},-p_{F}[X, Y]=\sharp_{\Lambda^{\prime \prime}}\{d[\sigma(X, Y)]\}
$$

for all the vector fields $X, Y \in \mathcal{V}_{p r}^{1,0}(M)$.

On the other hand we have

Proposition 5.5 The $\mathcal{F}$-coupling of the third kind pair $(\Lambda, E)$ is a Jacobi structure iff $M$ is a locally product manifold between the foliation $\mathcal{F}$ and a foliation $\mathcal{H}$ and, with respect to this locally product structure, $\Lambda^{\prime \prime}=0$ and $\left(\Lambda^{\prime}, E\right)$ is a Jacobi structure on $M$ such that its restrictions to the leaves of $\mathcal{H}$ are contact structures of these leaves.

Proof. The third kind coupling conditions imply the existence of a $(1,0)$ form $\xi \in k e r \sharp_{\Lambda^{\prime}}$ such that $\xi(E)=1$. Then, if we take $\alpha=\xi$ in the third condition (103), we get $\Lambda^{\prime \prime}=0$, and we remain with the first condition (103) and with

$$
\lambda\left(\left[\#_{\Lambda^{\prime}} \alpha, \sharp_{\Lambda^{\prime}} \beta\right]\right)=0, L_{E} \Lambda^{\prime}=0 \text {. }
$$

This implies the integrability of the normal distribution $H$, which provides the foliation $\mathcal{H}$, and shows that $\left(\Lambda^{\prime}, E\right)$ is the required Jacobi structure. Q.e.d.

Remark 5.1 Proposition 5.5 may be applied to a tubular neighborhood of a contact characteristic leaf of an arbitrary Jacobi manifold $(M, \Lambda, E)$ (see Example 5.1) and it tells us that all the neighboring characteristic leaves also are contact manifolds. This fact may be seen straightforwardly as follows. The characteristic leaf $L_{x_{0}}$ through the point $x_{0} \in M$ is a contact leaf iff there exists a 1 -form $\theta \in \operatorname{ann}\left(i m \sharp_{\Lambda}\right)$ such that $\theta_{x_{0}}\left(E_{x_{0}}\right) \neq 0$, and, if this condition holds at $x_{0}$, it holds on an open neighborhood of $x_{0}$. The situation 
is different for a locally conformal symplectic leaf $L_{x_{0}}$. For instance, take $M=\mathbb{R}^{4}=\{(q, p, u, t)\}$,

$$
\Lambda=u \frac{\partial}{\partial q} \wedge \frac{\partial}{\partial p}+\left(t \frac{\partial}{\partial t}\right) \wedge\left(p \frac{\partial}{\partial p}\right), \quad E=t \frac{\partial}{\partial t},
$$

and $x_{0}(q=1, p=0, u=1, t=0)$. Then, $L_{x_{0}}$ is 2-dimensional but, any neighboring leaf $L_{x_{1}}$ where $x_{1}(q=1, p=0, u=1, t \neq 0)$ is 3-dimensional, hence, a contact leaf.

It is interesting to discuss the coupling conditions for the transitive Jacobi structures. i.e., locally conformal symplectic structures and contact structures [2, 14].

Recall that a locally conformal symplectic structure of a differentiable manifold $M$ is a non degenerate 2 -form $\omega$, which satisfies the condition

$$
d \omega=\epsilon \wedge \omega,
$$

where $\epsilon$ is a closed 1-form (the Lee form). Then, the bivector field $\Lambda$ defined by $\sharp_{\Lambda}=-b_{\omega}^{-1}$ and the vector field $E=\sharp_{\Lambda} \epsilon$ define a Jacobi structure on $M$. In the general case of a manifold $M$ endowed with a pair $(\omega, \epsilon)$ where $\omega$ is a non degenerate 2-form and $\epsilon$ is a 1 -form, a pair $(\Lambda, E)$ can be defined similarly, and it may be coupling of either the first or the second kind for a foliation $\mathcal{F}$. This happens iff the pullback of $\omega$ to $\mathcal{F}$ is non degenerate, and then $H=\sharp_{\Lambda}($ ann $F)$ is the $\omega$ orthogonal distribution of $F$, and $E$ is always tangent or nowhere tangent to $\mathcal{F}$, respectively. In these cases, we also attribute the coupling property to the pair $(\omega, \epsilon)$ itself. Accordingly, if we want the coupling property in the locally conformal symplectic case, $\omega$ must be of the form (52) with respect to the bigrading defined by the symplecticorthogonal decomposition $T M=H \oplus F$ and satisfy (106). The result is

\section{Proposition 5.6 Let}

$$
\omega=\sigma_{2,0}+\theta_{0,2}
$$

be a non degenerate $\mathcal{F}$-coupling 2 -form on $\left(M^{2 n}, \mathcal{F}^{2 s}\right)$. If the coupling is of the first kind $(E \in \Gamma F)$, $\omega$ is locally conformal symplectic iff

$$
d^{\prime} \sigma=0, d^{\prime} \theta=0, d^{\prime \prime} \sigma+\partial \theta=\epsilon \wedge \sigma, d^{\prime \prime} \theta=\epsilon \wedge \theta
$$

If the coupling is of the second kind, $\omega$ is locally conformal symplectic iff

$$
d^{\prime} \sigma=\epsilon \wedge \sigma, d^{\prime \prime} \sigma+\partial \theta=0, d^{\prime} \theta=\epsilon \wedge \theta, d^{\prime \prime} \theta=0
$$


Proof. The Lee form $\epsilon$ has bidegree $(0,1)$ in the first case, and $(1,0)$ in the second. The results follow by equating the corresponding homogeneous components of the two sides of (106). Q.e.d.

In the first kind coupling case, the leaves of $\mathcal{F}$ get the induced locally conformal symplectic structure $(\theta, \epsilon)$ and in the second kind coupling case the leaves get the induced symplectic structure $\theta$. If the forms $\left(\epsilon_{0,1}, \theta_{0,2}\right)$, which define leafwise locally conformal symplectic structures of the leaves of $\mathcal{F}$, and the normal bundle of $\mathcal{F}$ are given, and we look for the form $\sigma$ that extends the given data to a locally conformal symplectic form $\omega$, we may encounter obstructions. In particular, $(M, \epsilon)(d \epsilon=0)$ has a twisted de Rham cohomology $H_{\epsilon}^{*}(M)$, which is that of the cochain complex $\left(\Omega^{*}(M), d_{\epsilon}=\right.$ $d-\epsilon \wedge)$. Using the degree decomposition of $d^{2}=0$, we see that the form $\partial \theta$ is $d_{\epsilon}$-closed, and the cohomology class $[\partial \theta] \in H_{\epsilon}^{3}(M)$ is an obstruction to the existence of $\sigma$. The same holds for a given pair $\left(\epsilon_{1,0}, \theta_{0,2}\right)$ which satisfies the last two conditions (108) and which we would like to extend to a locally conformal form $\omega$ on $M$.

A contact form $\phi$ on a differentiable manifold $M^{2 n+1}$ is a 1-form such that $\phi \wedge(d \phi)^{n} \neq 0$ at each point of $M$. The contact form $\phi$ defines the field of contact hyperplanes $\pi$ of equation $\phi=0$ and the Reeb vector field $E$ characterized by

$$
\phi(E)=1, i(E) d \phi=0
$$

and we may write

$$
T M=\pi \oplus \operatorname{span}\{E\}, T^{*} M=\pi^{*} \oplus \operatorname{span}\{\phi\} .
$$

The mapping $b_{d \phi}$ is an isomorphism of $\pi$ onto $\pi^{*}$, and we may define a bivector field $\Lambda$ by asking $\sharp_{\Lambda}$ to be zero on $\operatorname{span}\{\phi\}$ and $-b_{d \phi}^{-1}$ on $\pi^{*}$. It turns out that the pair $(\Lambda, E)$ is a Jacobi structure [2, 14.

Let $(M, \phi)$ be a contact manifold. We will look at two kinds of foliations $\mathcal{F}$ of $M$ : foliations of the contact type, characterized by the condition $E \in \Gamma F$ $(F=T \mathcal{F})$, and foliations of the symplectic type, characterized by $F \subseteq \pi$.

If $\mathcal{F}$ is of the contact type, only coupling of the first kind can occur. More exactly, if $E \in \Gamma F, F$ and $\pi$ are transversal, $F \cap \pi$ is a vector subbundle of $T M$, ann $F \subseteq \pi^{*}$ and $b_{d \phi}^{-1}($ ann $F)=(F \cap \pi)^{\perp_{d \phi}}$. The coupling condition holds iff $d \phi$ is non degenerate on $(F \cap \pi)^{\perp_{d \phi}}$, equivalently, $d \phi$ is non degenerate on $(F \cap \pi)$. This means that the Jacobi structure defined by the contact form $\phi$ is coupling for $\mathcal{F}$ iff the pullbacks of $\phi$ to the leaves of $\mathcal{F}$ are contact forms 
of the leaves. The corresponding normal bundle $H$ of $\mathcal{F}$ is $(F \cap \pi)^{\perp_{d \phi}}$. The problem of extending a smooth family of contact structures of the leaves of $\mathcal{F}$ to a coupling contact structure of $M$ (in the sense of Jacobi structures) was recently studied for fiber bundles by Lerman [5].

If $\mathcal{F}$ is of the symplectic type, $\operatorname{ann}(F \oplus E)=(\operatorname{ann} F) \cap \pi^{*}$ and $b_{d \phi}^{-1}[(\operatorname{ann} F) \cap$ $\left.\pi^{*}\right]=F^{\perp_{d \phi}} \subseteq \pi$, which does not contain $E$. Accordingly, only couplings of the third kind might occur, and the Jacobi structure defined by the contact form $\phi$ would be coupling for $\mathcal{F}$ iff the pullbacks of $d \phi$ to the leaves of $\mathcal{F}$ would be symplectic forms of the leaves. This is impossible since $d \phi=0$ along any integral submanifold of the contact distribution $\pi$.

We end this paper by indicating some possible applications of the VorobievPoisson structures (Section 4) to Jacobi manifolds.

First we recall that, if $(M, \Lambda, E)$ is a Jacobi manifold, the jet bundle $J^{1}(M, \mathbb{R}) \approx T^{*} M \oplus \mathbb{R}$ has a natural structure of a Lie algebroid, induced by the restriction to $M \approx M \times\{0\}$ of the cotangent Lie algebroid of the Poisson manifold $(M \times \mathbb{R}, P)$ with $P$ given by (92) (e.g., see [14]). The corresponding Lie bracket is

$$
\begin{gathered}
\{(\alpha, f),(\beta, g)\}=\left(\{\alpha, \beta\}_{\Lambda}+f L_{E} \beta-g L_{E} \alpha\right. \\
-\alpha(E) \beta+\beta(E) \alpha,\{f, g\}-\Lambda(d f-\alpha, d g-\beta)),
\end{gathered}
$$

where $\alpha, \beta \in \Omega^{1}(M), f, g \in C^{\infty}(M)$, the bracket of functions is (90) and the $\Lambda$-bracket of 1 -forms is (5). the The corresponding anchor map is

$$
\rho(\alpha, f)=\sharp_{\Lambda} \alpha+f E .
$$

Since the characteristic leaves of the Jacobi structure are integral submanifolds of the distribution $\operatorname{span}\left\{i m \sharp_{\Lambda}, E\right.$, the bracket (111) actually computes along these leaves and, for any characteristic leaf $S$ of $(\Lambda, E)$, $\left.J^{1}(M, \mathbb{R})\right|_{S}$ is a transitive Lie algebroid with the basis $S$.

Furthermore, we have

Proposition 5.7 For any characteristic leaf $S$, the kernel $K=$ ker $\rho$ of the anchor map (112) restricted to $S$ is isomorphic with the annihilator ann $_{M \times \mathbb{R}}(T S)$ of $S$ seen as the submanifold $S \times\{0\}$ of $M \times \mathbb{R}$.

Proof. If the dimension of $S$ is even $S$ is a locally conformal symplectic manifold, $T S=i m \sharp_{\left.\Lambda\right|_{S}}$, and there exist 1-forms $\left.\epsilon \in T^{*} M\right|_{S}$ such that $\left.E\right|_{S}=$ $\sharp_{\Lambda}(\epsilon)$. As in formula (92), we denote by $t$ the coordinate on $\mathbb{R}$. The mapping 
$(\alpha, f) \mapsto(\alpha+f \epsilon)+f d t$ yields an isomorphism $K \rightarrow a n n_{M \times \mathbb{R}}(T S)$ as required, which is not unique since it depends on the choice of $\epsilon$.

If the dimension of $S$ is odd $S$ is a contact manifold with the Reeb vector field $\left.E\right|_{S}$, which neither vanishes nor belongs to $i m \sharp_{\Lambda}$ at each point of $S$. Accordingly, (112) yields $K=k e r \sharp_{\Lambda}$ along $S$. On the other hand, $a n n_{M}(T S)=$ $\left(k e r \sharp_{\Lambda}\right) \cap \operatorname{ann}(E)$. Hence, if we choose the 1-form $\xi \in \Gamma\left(k e r \sharp_{\Lambda}\right)$ such that $\xi(E)=1$ along $S$, we get an isomorphism $K \approx \operatorname{ann}_{M}(T S) \oplus \operatorname{span}\{\xi\}$, which allows us to represent the elements of $K$ under the form $\beta+h \xi$ where $\beta \in \operatorname{ann}_{M}(T S), h \in C^{\infty}(M)$. Furthermore, the mapping $(\beta+h \xi, 0) \mapsto \beta+h d t$ yields an isomorphism $K \rightarrow a n n_{M \times \mathbb{R}}(T S)$ as required. Again this isomorphism is not unique since it depends on the choice of $\xi$. Q.e.d.

Now, assume that $S$ is an embedded characteristic leaf of the Jacobi manifold $(M, \Lambda, E)$ and that the induced structure of $S$ is globally conformal symplectic, i.e., there exists on $S$ a symplectic form $\omega$ and a function $f \in$ $C^{\infty}(M)$ such that the Jacobi structure of $S$ is defined by the 2 -form $e^{f} \omega$. The symplectic form $\omega$ allows us to define a Vorobiev-Poisson structure $\Pi$ on a neighborhood $U$ of $S$ seen as the zero section of the total space of the Lie coalgebras bundle $K^{*}$. By Proposition [5.7 $U$ may also be seen as a tubular neighborhood of $S \times\{0\}$ defined by a normal bundle of this submanifold in $M \times\{\mathbb{R}\}$. Using formula (97) with $a=e^{f}, \Lambda=\Pi, E=0$ we get a Jacobi structure on $U$ for which $S$ is a characteristic leaf with the same induced Jacobi structure as the one induced by the original $(\Lambda, E)$. Notice that we cannot just use Vorobiev's construction along a locally conformal symplectic leaf since the form (64) associated to a locally conformal symplectic form $\omega$ does not satisfy conditions (105).

On the other hand, if $S$ is a contact, embedded, characteristic leaf of the Jacobi manifold $(M, \Lambda, E), S \times\{\mathbb{R}\}$ is a symplectic leaf of the Poisson structure $P$ of $M \times\{\mathbb{R}\}$ and its symplectic structure is the so-called symplectification of the contact structure of $S$. Using this symplectic structure, we get a Vorobiev-Poisson structure on a neighborhood of the zero section of a normal bundle $N(S \times\{\mathbb{R}\})$ in $M \times\{\mathbb{R}\}$. This neighborhood may be seen as having the form $V \times\{\mathbb{R}\}$ where $V$ is a neighborhood of $S$ seen as the zero section of a normal bundle $N S$ of $S$ in $M$. Therefore, $S$ has a neighborhood $V \subseteq N S$ which is a hypersurface of a Vorobiev-Poisson manifold.

Remark 5.2 If $L_{x_{0}}$ is a locally conformal symplectic leaf of $(M, \Lambda, E)$ with the 2 -form $\omega$ and the (closed) Lee form $\epsilon$, the symplectic leaf of $(M \times \mathbb{R}, P)$ through $\left(x_{0}, 0\right)$ is the hypersurface of $\left(L_{x_{0}} \times \mathbb{R}, P\right)$, which integrates the 
equation $\epsilon-d t=0$, and passes through $x_{0}$.

\section{References}

[1] I. M. Gelfand and I. Ya. Dorfman, The Schouten bracket and Hamiltonian operators. Funkt. Anal. Prilozhen. 14 (3) (1980), 71-74.

[2] P. Dazord, A. Lichnerowicz and Ch.-M. Marle, Structures locales des variétés de Jacobi, J. Math. pures et appl., 70 (1991), 101-152.

[3] V. Guillemin, E. Lerman and S. Sternberg, Symplectic fibrations and multiplicity diagrams. Cambridge Univ. Press, Cambridge, 1996.

[4] D. Iglesias and J. C. Marrero, Some linear Jacobi structures on vector bundles, C. R. Acad. Sci. Paris, Sér. I Math., 331 (2000), 125-130.

[5] E. Lerman, Contact fiber bundles, arXiv:math.DG/0301137.

[6] A. Lichnerowicz, Global theory of connections and holonomy groups. Noordhoof Intern. Publ., Leyden, 1976.

[7] K. C. H. Mackenzie, Lie Groupoids and Lie Algebroids in Differential Geometry, LMS Lecture Notes Ser., Vol. 124, Cambridge Univ. Press, Cambridge, 1987.

[8] K. C. H. Mackenzie and P. Xu, Lie bialgebroids and Poisson groupoids, Duke Math. J., 18 (1994), 415-452.

[9] P. Molino, Riemannian foliations. Progress in Math. 73, Birkhäuser, Boston, 1988.

[10] R. Montgomery, J. E. Marsden and T. Raţiu, Gauged Lie-Poisson structures, Cont. Math. AMS, Vol. 28 (Boulder Proceedings on Fluids and Plasmas), 1984, 101-114.

[11] S. Sternberg, On minimal coupling and the symplectic mechanics of a classical particle in the presence of a Yang-Mills field, Proc. Nat. Acad. Sci. USA, 74 (1977), 5253-5254.

[12] I. Vaisman, Cohomology and differential forms. M. Dekker, Inc., New York, 1973. 
[13] I. Vaisman, Lectures on the Geometry of Poisson Manifolds. Progress in Math. 118, Birkhäuser, Basel, 1994.

[14] I. Vaisman, A lecture on Jacobi manifolds. Selected topics in Geom. and Math. Physics, 1 (2002), 81-100.

[15] I. Vaisman, Hamiltonian structures on foliations, J. Math. Physics, 43 (2002), 4966-4977.

[16] Y. Vorobjev, Coupling tensors and Poisson geometry near a single symplectic leaf. In: Lie algebroids and related topics in differential geometry (J. Kubarski, P. Urbański and R. Wolak, eds.), Banach Center Publ., Vol. 54, Warszawa, 2001, p. 249-274.

Department of Mathematics

University of Haifa, Israel

E-mail: vaisman@math.haifa.ac.il 